\title{
Cuando la conservación no puede seguir el ritmo del desarrollo: Estado de salud de los ecosistemas coralinos del Pacífico Norte de Costa Rica
}

\author{
Juan José Alvarado ${ }^{1,2,3}$, Andrés Beita-Jiménez ${ }^{2}$, Sebastián Mena ${ }^{2}$, Cindy Fernández-García ${ }^{1,2,4}$, \\ Jorge Cortés ${ }^{1,2,3}$, Celeste Sánchez-Noguera ${ }^{1,5}$, Carlos Jiménez ${ }^{1,6}$ \& Ana Gloria Guzmán-Mora ${ }^{7}$ \\ 1. Centro de Investigación en Ciencias del Mar y Limnología, Universidad de Costa Rica; juanalva76@yahoo.com; \\ cindy.fernandezgarcia@ucr.ac.cr; jorge.cortes@ucr.ac.cr \\ 2. Escuela de Biología, Universidad de Costa Rica; abeitaj@gmail.com; sebasmenago@gmail.com \\ 3. Museo de Zoología, Universidad de Costa Rica \\ 4. Herbario de la Universidad de Costa Rica \\ 5. Leibniz Center for Tropical Marine Ecology, Bremen, Germany; celeste08@gmail.com \\ 6. The Cyprus Institute Nicosia, Cyprus, c.jimenez@cyi.ac.cy \\ 7. Conservación Internacional; aguzman@conservation.org
}

Recibido 10-IV-2017. Corregido 10-I-2017. Aceptado 08-II-2018.

\begin{abstract}
When conservation can keep up with development's pace: Health status of coral ecosystems in the North Pacific of Costa Rica. Coral reefs are diverse and productive ecosystems, despite this, they are being threatened by human activities that enhance the detrimental impact of the natural phenomenon's like Harmful Algal Blooms (HAB). The north Pacific of Costa Rica has been characterized as one of the best regions for the coral reefs development in the country. However, many of these ecosystems are being lost as a result of eutrophication, overfishing, invasive species and others impacts that affect the region. In the present study, live coral cover in the north Pacific was $5.0 \pm 10.4$ (s.d.) \%, with a domination in the ecosystems by turf algae. Twenty-six invertebrate taxa were registered in the region with the predominance of the sea urchin Diadema mexicanum. Ninety-four species of reef fish were identified. Snappers and some planktivores species were the groups with the highest frequency and abundance. The localities previously studied in the 1990 decade, presented a mean live coral cover between $40-50 \%$, whereby the actual state of the reef reflect a significant deterioration. This decline in coral cover is due to natural events like the El Niño, as well as the decrease in water quality in the region. In recent years, proliferations of the invasive seaweed Caulerpa sertularioides and high densities of bioerosive sea urchins have been reported, mainly associated with HAB events. Likewise, fish communities have low biomass, especially in the vicinity of fishing villages. The state of the reefs in the North Pacific is worrying and requires actions for its recovery and conservation, for which there must be better planning of the development of projects and activities on the coast. Rev. Biol. Trop. 66(Suppl. 1): S280-S308. Epub 2018 April 01.
\end{abstract}

Key words: Harmful Algal Blooms, invasive species, coastal alteration, El Niño, overfishing.

Los corales son pequeños organismos coloniales de anatomía simple, pero con una incalculable importancia ecológica en las costas tropicales de todo el planeta. Estos organismos son los principales constructores de arrecifes coralinos, el ecosistema más diverso del océano, solo comparado con los bosques tropicales (Reaka-Kudla, 1997). En los arrecifes coralinos se encuentran presentes casi todos los phyla existentes, así como las más sorprendentes y complejas interacciones ecológicas (Birkeland, 1997; Reaka-Kudla, 1997; Karlson, 1999). Lamentablemente, hoy en día estos afrontan serias amenazas producto de las actividades antrópicas, por ejemplo la explotación sin regulación de sus recursos, la sobrepesca, la contaminación y la eutroficación, que potencializan fenómenos naturales como las 
Floraciones Algales Nocivas (FAN) (Guzman, Cortés, Glynn, \& Richmond, 1990; MoralesRamírez, Víquez, Rodríguez, \& Vargas, 2001; Jiménez, 2007).

El Pacífico Norte de Costa Rica (Península de Santa Elena, Golfo de Papagayo y Península de Nicoya) (Fig. 1) se considera dentro una de las regiones con desarrollo importante de arrecifes coralinos del país (Cortés, \& Jiménez, 2003), pese a que presenta condiciones físicas naturales que restringen el desarrollo coralino (Cortés, 1997). Las primeras investigaciones en esta región indican que los arrecifes estaban dominados por colonias ramificadas de los corales Pocillopora damicornis y Pocillorpora elegans, con coberturas promedio de coral vivo de hasta $44 \%$, con extensiones de $2 \mathrm{~km}$ de largo y una diversidad de 16 especies constructoras de arrecifes coralinos (Glynn, Druffel, \& Dunbar, 1983; Cortés, 1996-1997a, b, 2016; Cortés, \& Guzman, 1998; Jiménez, 2001a, b; Cortés, \& Jiménez, 2003; Jiménez, Bassey, Segura, \& Cortés, 2010). Bahía Culebra ha sido la localidad de mayor estudio de esta región, y en ella se describieron la presencia de especies poco comunes de coral como Cycloseris curvata, Leptoseris papyracea y Pocillopora meandrina (Jiménez, 2001a; Cortés, \& Jiménez, 2003; Cortés, Jiménez, Fonseca, \& Alvarado, 2010).

En el Pacífico Norte, de diciembre a abril, ocurre un fenómeno de surgencia por efectos de los vientos alisios, que produce bajas temperaturas, bajos valores de $\mathrm{pH}$, y altas concentraciones de nutrientes, lo que convierte a la región en una zona muy productiva (Alfaro et al., 2012; Fernández-García, Cortés, Alvarado, \& Nivia-Ruiz, 2012; Rixen, Jiménez, \& Cortés, 2012; Cortés, 2014; Studldreier et al., 2015a). Además de esta entrada natural de nutrientes, hay eutroficación de origen antropogénico durante la época lluviosa (mayo-noviembre), por efecto de las escorrentías (FernándezGarcía et al., 2012; Studldreier et al., 2015a, $\mathrm{b}, \mathrm{c})$. Lo que ha producido deterioro paulatino de la calidad del agua, dando origen a eventos de FAN (Morales-Ramírez et al., 2001; Vargas-Montero, \& Freer, 2004; Jiménez, 2007;
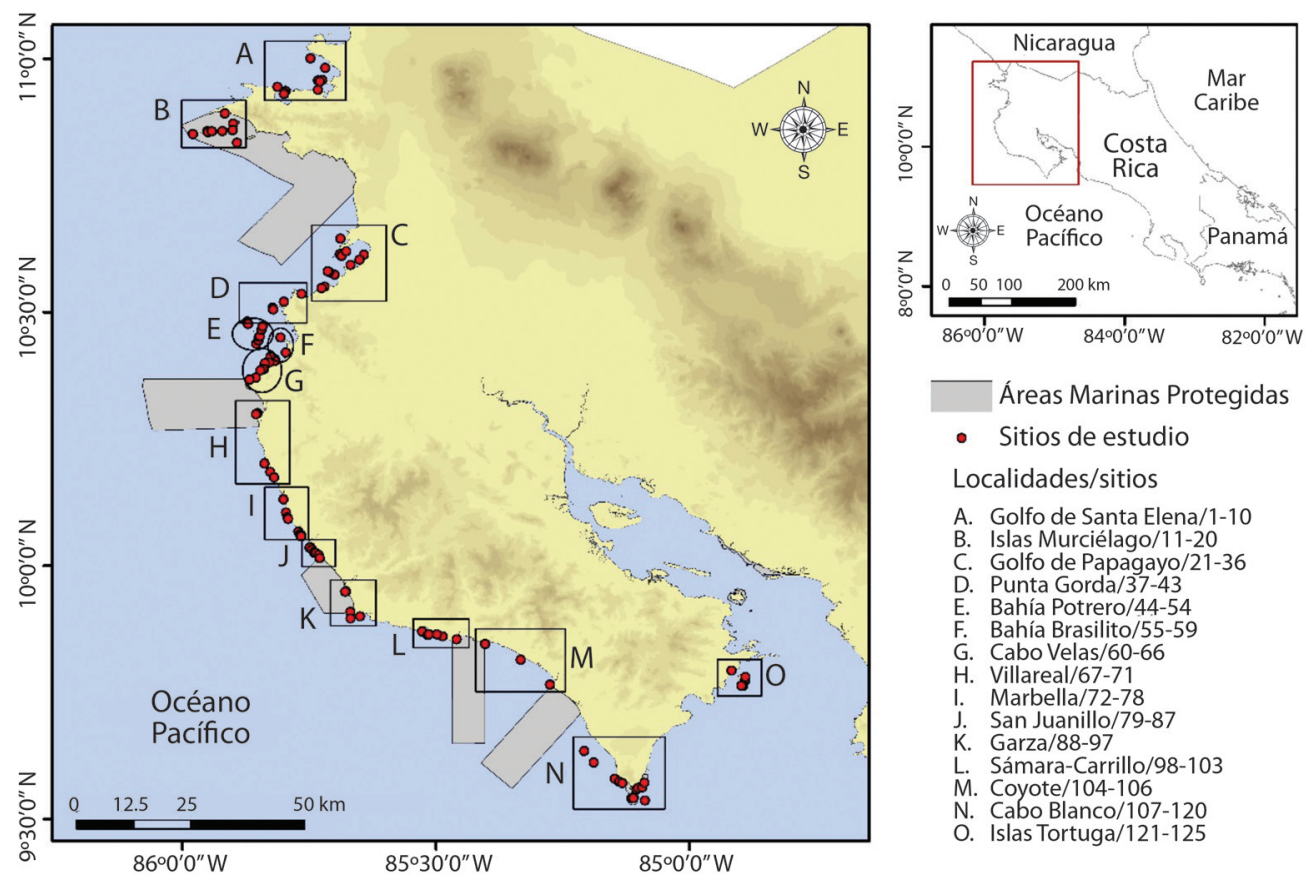

Áreas Marinas Protegidas

- Sitios de estudio Localidades/sitios

A. Golfo de Santa Elena/1-10

B. Islas Murciélago/11-20

C. Golfo de Papagayo/21-36

D. Punta Gorda/37-43

E. Bahía Potrero/44-54

F. Bahía Brasilito/55-59

G. Cabo Velas/60-66

H. Villareal/67-71

I. Marbella/72-78

j. San Juanillo/79-87

K. Garza/88-97

L. Sámara-Carrillo/98-103

M. Coyote/104-106

N. Cabo Blanco/107-120

O. Islas Tortuga/121-125

Fig. 1. Localidades y sitios de estudio en el Pacífico Norte, Costa Rica.

Fig. 1. Localities and sampling sites on the North Pacific, Costa Rica. 
Vargas-Montero, Freer-Bustamante, Guzmán, \& Vargas, 2008; Calvo-Vargas, Berrocal-Artavia, \& Abarca, 2016). En muchos casos, estos FAN producen el deterioro de los fondos rocosos, causando blanqueamiento y mortalidad coralina (Jiménez, 2007). Sobre el sustrato coralino muerto se propicia el desarrollo de tapetes algales ("turf"), lo que probablemente favorece el crecimiento explosivo de poblaciones de erizos de mar que, a su vez, incrementó su impacto bioerosivo sobre los arrecifes (Alvarado, Cortés, \& Reyes-Bonilla, 2012a). Por otro lado, el deterioro de la calidad del agua marina facilita también al alga invasiva Caulerpa sertularioides, que sobrecrece los corales y modifica el fondo marino (Fernández, \& Cortés 2005; Fernández-García et al., 2012).

Este trabajo pretende describir el cambio que han sufrido los arrecifes coralinos en el Pacífico Norte de Costa Rica, relacionado con los diferentes impactos que ha sufrido la zona como El Niño, desarrollo costero y la presencia de organismos invasores y bioerosionadores. Algunos de los sitios evaluados no poseen línea base de cobertura y son descritos por primera vez en esta investigación. Las comparaciones cuantitativas realizadas se basan en evaluaciones previas completadas para Bahía Culebra, mientras que para los otros sitios, las comparaciones son cualitativas con base en características de los arrecifes descritas en publicaciones previas (Cortés, 1996-1997b, 2016; Cortés, \& Jiménez, 2003).

\section{MATERIALES Y MÉTODOS}

Cobertura del fondo arrecifal: Para evaluar la cobertura de los fondos arrecifales, se visitaron 125 sitios a lo largo del Pacífico Norte de Costa Rica (Fig. 1). Los sitios se dividieron en 15 localidades (identificadas de A-O), para facilitar las comparaciones geográficas. Los datos de cobertura provienen de diferentes periodos entre 2009 y 2017, y fueron recolectados utilizando distintas metodologías de muestreo.

Las evaluaciones de las localidades A (mayo 2012), C (octubre 2010), D (octubre
2010), E (agosto y octubre 2010), F (julio y agosto 2009), G (enero 2011), H (enero 2010), I (enero 2010), J (febrero 2010), K (enero 2010) y N (marzo 2012) (Fig. 1) se realizaron mediante transectos lineales con cadena (Jiménez, 2001a, b; Jiménez, Cortés, León, \& Ruiz, 2001, et al., 2010). El método consiste en extender una cadena liviana (10 $\mathrm{m}$ de longitud, eslabones de $\approx 1 \mathrm{~cm}$ ), siguiendo el contorno de la superficie del fondo, y registrando el número de eslabones que coinciden con las categorías evaluadas (coral vivo, coral muerto, coral blanqueado, alga calcárea costrosa, macroalga, turf, arena y cascajo). Las sumatorias totales obtenidas por categoría se convierten a porcentajes de cobertura. En cada sitio de muestreo se efectuaron cinco réplicas, obteniendo una longitud total de $50 \mathrm{~m}$.

Las localidades B (octubre 2014), C (julio 2014), J (julio 2013), L (julio 2013 y febrero 2014), M (julio 2013) y O (abril 2013 y febrero 2014) se evaluaron mediante transectos de 10 $\mathrm{m}$ de longitud, con cuadrículas de $1 \mathrm{~m}^{2}$, subdividida en cuadrantes de $0.01 \mathrm{~m}^{2}$ (Weinberg, 1981). En estas localidades se realizaron entre tres y seis transectos por cada sitio visitado (Alvarado, Beita, Mena, Fernández-García, \& Guzmán-Mora, 2015, 2016a).

Macroinvertebrados móviles: Los macroinvertebrados fueron cuantificados en las localidades: B, C, D, J, L, M, O. En el resto de localidades no se evaluó la composición de estos organismos. Sobre los transectos de cobertura de fondo se muestreó $1 \mathrm{~m}$ a cada lado, entre grietas y rocas sin perturbar el ambiente, para un área total de $20 \mathrm{~m}^{2}$, en los que se cuantificaron todos los macroinvertebrados conspicuos de más de $2.5 \mathrm{~cm}$. En las Islas Murciélago se utilizó un muestreo invasivo, se registraron algunos organismos bajo rocas. Las categorías utilizadas fueron moluscos (bivalvos, cefalópodos y gasterópodos, principalmente opistobranquios), crustáceos (langostas y cangrejos flecha) y equinodermos (pepinos de mar, erizos de mar y estrellas de mar). Se identificaron los organismos a nivel de género 
y especie en la medida de lo posible (Alvarado et al., 2015, 2016a).

Con el fin de determinar cuáles macroinvertebrados son los predominantes en la zona de estudio, se realizó un análisis de dominancia específica basado en la densidad relativa y la ocurrencia (cantidad de sitios donde fue observado el taxón). Se consideró el promedio de ambos parámetros como límite para definir las categorías: especie predominante (densidad y ocurrencia altas), especie ocasional (densidad alta y ocurrencia baja), especie común (densidad baja y ocurrencia alta) y especie rara (densidad y ocurrencia bajas). Este análisis no incluyó los datos de macroinvertebrados móviles registrados en las Islas Murciélago, debido a que la metodología aplicada para su cuantificación fue distinta.

Se calculó la riqueza de especies (S), el índice de heterogeneidad de Shannon ( ${ }^{\prime}$ $(\log 10))$ y el índice de diferenciación taxonómica $\left(\Delta^{*}\right)$ para cada sitio. Para el $\Delta^{*}$ se utilizaron seis niveles taxonómicos con sus respectivos valores: especies $(\omega=$ pesos $) \omega=$ 16.66 (especies en el mismo género), genero $\omega=33.33$ (misma familia, pero géneros diferentes), familia $\omega=50.00$ (mismo orden, pero familia diferente), orden $\omega=66.66$ (misma clase, pero orden diferente), clase $\omega=83.33$ (mismo filo, pero clase diferente) y filo $\omega=100$ (filos diferentes).

Con el fin de cuantificar la similitud de composición entre las localidades de estudio, se realizó el índice de Bray-Curtis, basado en matriz de abundancia, y se ordenó con un análisis de escalamiento multidimensional no métrico (nMDS).). Los datos de densidad promedio (ind $\mathrm{m}^{-2}$ ) por sitio se estandarizaron $\mathrm{y}$ se transformaron con la función $\log _{10}(\mathrm{X}+1)$. Posteriormente, se determinó si la composición de macroinvertebrados difiere entre sitios por localidad a través del análisis de similitud de una vía (ANOSIM). A su vez, se realizó el análisis de similitud porcentual (SIMPER) para cuantificar las especies que contribuyeron más a las diferencias entre localidades. Para estos análisis se utilizó el programa PRIMER 6.1.4.
Peces arrecifales: En los mismos transectos donde se determinó la cobertura del fondo y la composición de macroinvertebrados móviles, se cuantificó la composición íctica. Para ello se contabilizó y estimó visualmente la talla de todos los peces observados en la línea del transecto, formando un túnel imaginario de $5 \mathrm{~m}$ de ancho y $5 \mathrm{~m}$ de alto $(10 \mathrm{~m}$ x $5 \mathrm{~m}$ x $5 \mathrm{~m})$. Se identificó la especie, estimó el tamaño de cada pez y se clasificaron en las siguientes categorías: $<5,5-10,10-15,15-20,20-25,25-50$, 50-100, 100-150, 150-200, 200-250, 250-300 $\mathrm{cm}$ (Alvarado et al., 2015, 2016b). Las frecuencias de tallas de cada especie se transformaron en biomasa usando los parámetros $a$ y $b$ obtenidos de FishBase (Froese, \& Pauly, 2016).

De la misma manera que con los macroinvertebrados móviles, se elaboró un análisis de dominancia específica basado en la densidad relativa y la ocurrencia, calculando la riqueza de especies (S), el índice de heterogeneidad de Shannon (H' $(\log 10)$ ) y el índice de diferenciación taxonómica $\left(\Delta^{*}\right)$ para cada sitio. Para determinar la similitud entre sitios en términos de la biomasa de peces ( $\mathrm{t} \mathrm{ha}^{-}$), se realizó el análisis de escalamiento multidimensional no métrico (nMDS) basados en la matriz de disimilitud de Bray-Curtis. Las variables fueron transformadas con la función $\log _{10}(X+1)$. Se determinó si las localidades explican el agrupamiento de los sitios mediante un ANOSIM. A su vez, se realizó el análisis de similitud porcentual (SIMPER) para cuantificar las especies que contribuyeron más a las diferencias entre localidades. Los análisis se llevaron a cabo utilizando el software PRIMER 6.1.4.

\section{RESULTADOS}

Cobertura del fondo arrecifal: La cobertura del fondo sin importar la fecha, localidad o el método de evaluación estuvo predominada por turf (Fig. 2), con coberturas que rondaron entre $13.2 \%$ y $85.7 \%$ (promedio: $61.1 \pm$ 19.8(d.e) \%). La única localidad que presentó una cobertura levemente mayor al turf fue Bahía Santa Elena, donde la cobertura promedio de coral vivo fue de $44.8 \%$ (Fig. 2). Para 


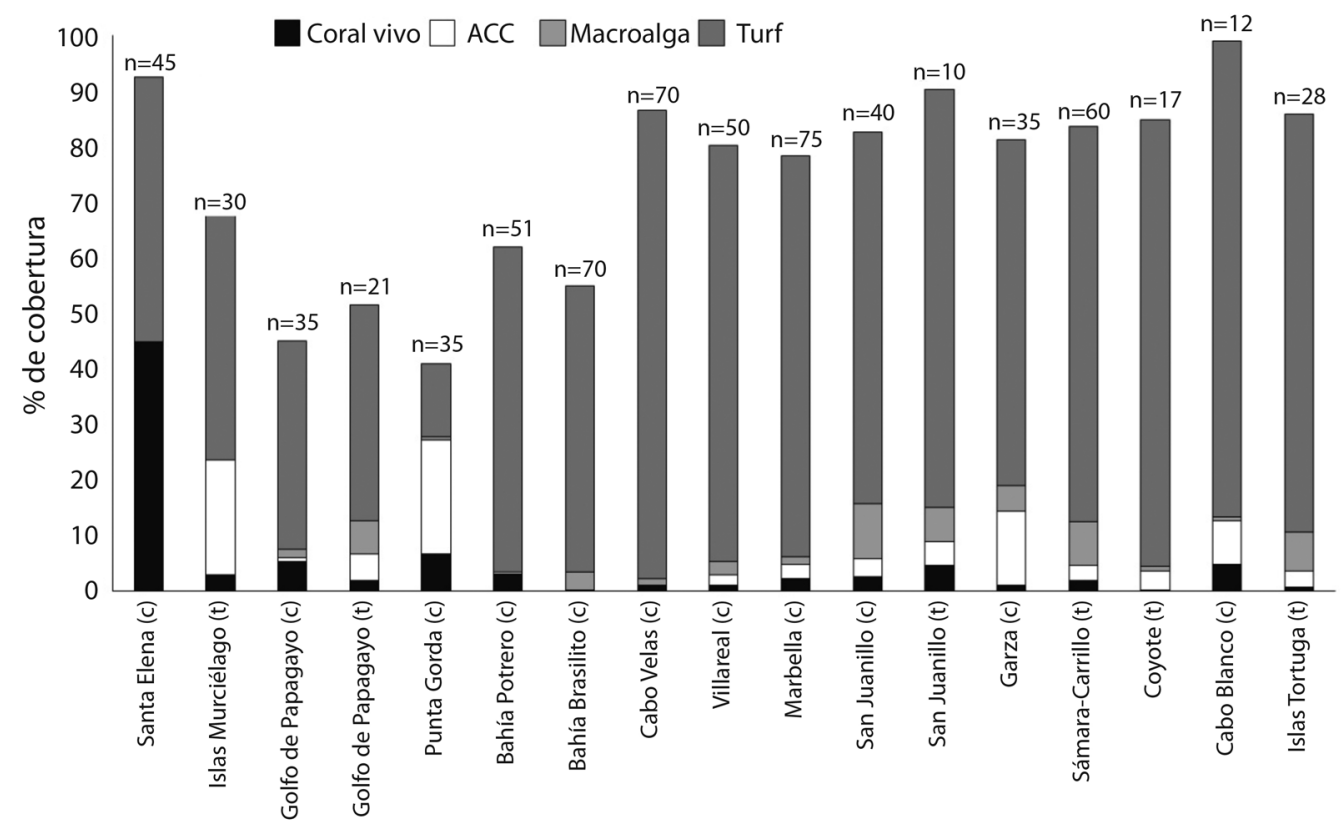

Fig. 2. Porcentaje (\%) de cobertura promedio del sustrato en las localidades de estudio en el Pacífico Norte, Costa Rica. c: transecto con cadena; $\mathrm{t}$ : transecto con cuadrante. n: número de transectos totales. ACC: Algas Calcáreas Costrosas.

Fig. 2. Percentage (\%) of average coverage of the substrate in the study localities in the North Pacific, Costa Rica. c: transect with chain; t: transect with quadrant. n: number of total transects. CCA: Croustouse Calcareous Algae.

la categoría de coral vivo, los valores rondaron entre $0.2 \%$ y $44.8 \%$, estando en promedio para el Pacífico Norte de $5.0 \pm 10.4$ (d.e) \%. Otras categorías de sustrato como las macroalgas o las algas calcáreas costrosas (ACC), tuvieron coberturas promedio bajas $3.1 \pm 3.2$ (d.e) $\%$ y $5.3 \pm 6.7$ (d.e) $\%$, respectivamente). El valor más alto de macroalgas se cuantificó en las localidades de San Juanillo (9.0\%), SamaraCarrillo (7.8 \%) y Golfo de Papagayo (6.0 \%). Por su parte las localidades con mayor porcentaje de ACC fueron las Islas Murciélago (20.7 \%) y Punta Gorda (20.5 \%) (Fig. 2).

Macroinvertebrados móviles: Utilizando el muestreo invasivo en la localidad B se registraron 26 taxa de macroinvertebrados (Anexo 1), mientras que en las localidades $\mathrm{C}, \mathrm{D}, \mathrm{J}$, L, M y O se observaron 38 taxa (Cuadro 1). En estas localidades, la mayoría de especies fueron predominantes (37\%), seguidas de raras $(32 \%)$, comunes $(18 \%)$ y en menor proporción ocasionales (13\%) (Fig. 3). Los taxa de macroinvertebrados que predominaron en la región fueron los erizos de mar Astropyga pulvinata, Diadema mexicanum, Echinometra vanbrunti y Eucidaris thouarsii; la estrella de mar Phataria unifascialis; los opistobranquios Elysia diomedea y Felimare agassizii; los bivalvos Hyotissa hyotis y Pinctada mazatlanica; el gasterópodo Vasum spp.; el pulpo Octopus spp.; y los crustáceos Panulirus gracilis y Stenorhynchus debilis.

Los sitios con mayor riqueza de especies (S) fueron Punta Carrillo, El Mortero, Isla Chora 1, El Muñeco y Punta el Indio (Cuadro 2). El sitio con la menor riqueza fue El Santuario con solo dos especies (P. unifacialis y Toxopneustes roseus). En cuanto a la composición de macroinvertebrados se presentó mayor heterogeneidad (H' $\left.\left(\log _{10}\right)\right)$ en Isla Chora 1 (1.05), Punta Carrillo (1.01), Punta El Indio (1.01), El Cambute (0.99) y El Muñeco (0.91). Los sitios con menor índice de Shannon (< 0.25 ) fueron sitios en las Islas Murciélago y Playa Blanca (Cuadro 2), donde se observó 
CUADRO 1

Especies de macroinvertebrados presentes en los sitios evaluados en el Pacífico Norte de Costa Rica

TABLE 1

Species of macroinvertebrates present on the evaluated sites in the North Pacific of Costa Rica

\begin{tabular}{|c|c|c|c|}
\hline \multicolumn{4}{|c|}{ Clasificación taxonómica } \\
\hline \multicolumn{2}{|c|}{ Filo Echinodermata } & 18 & Hyotissa hyotis \\
\hline \multicolumn{2}{|c|}{ Clase Asteroidea } & 19 & Pinctada mazatlanica \\
\hline 1 & Nidorellia armata & 20 & Pinna rugosa \\
\hline 2 & Pharia pyramidata & 21 & Spondylus limbatus \\
\hline 3 & Phataria unifascialis & 22 & Spondylus spp. \\
\hline \multicolumn{2}{|c|}{ Clase Echinoidea } & \multicolumn{2}{|c|}{ Clase Cephalopoda } \\
\hline 4 & Astropyga pulvinata & 23 & Octopus spp. \\
\hline 5 & Diadema mexicanum & \multicolumn{2}{|c|}{ Clase Gastropoda } \\
\hline 6 & Echinometra vanbrunti & 24 & Fasciolariidae spp. \\
\hline 7 & Eucidaris thouarsii & 25 & Hexaplex princeps \\
\hline 8 & Toxopneustes roseus & 26 & Lobatus galeatus \\
\hline 9 & Tripneustes depressus & 27 & Murex spp. \\
\hline \multicolumn{2}{|c|}{ Clase Holothuroidea } & 28 & Muricidae spp. \\
\hline 10 & Holothuria (Halodeima) atra & 29 & Vasum spp. \\
\hline 11 & Holothuria (Selenkothuria) lubrica & \multicolumn{2}{|c|}{ Infraclase Opistobranchia } \\
\hline 12 & Isostichopus fuscus & 30 & Doriprismatica sedna \\
\hline 13 & Psolidae spp. & 31 & Elysia diomedea \\
\hline \multicolumn{2}{|c|}{ Filo Arthropoda } & 32 & Elysia spp. \\
\hline \multicolumn{2}{|c|}{ Orden Decapoda } & 33 & Felimare agassizii \\
\hline 14 & Panulirus gracilis & 34 & Felimida dalli \\
\hline 15 & Stenorhynchus debilis & 35 & Felimida sphoni \\
\hline \multicolumn{2}{|c|}{ Filo Mollusca } & 36 & Flabellina cynara \\
\hline \multicolumn{2}{|c|}{ Clase Bivalvia } & 37 & Flabellina marcusorum \\
\hline 16 & Atrina spp. & 38 & Opisthobranchia spp. \\
\hline 17 & Chama spp. & & \\
\hline
\end{tabular}

alta densidad de D. mexicanum (Anexo 1). Los sitios con mayor distintividad taxonómica $\left(\Delta^{*}\right)$ fueron Esmeralda (100), Tiburón (95), Bahía Curú (93), El Mortero (92) y Matapalo (90). En los sitios de las Islas Murciélago se observaron los valores más bajos de $\Delta^{*}$ (Cuadro 2 ), donde se registró gran cantidad de equinodermos (Anexo 1).

En cuanto a la similitud de las localidades por composición de especies, se conforman cinco grupos, donde se agruparon los sitios de Sámara-Carrillo con los sitios de Islas Tortuga. Las comunidades de invertebrados estudiadas en Coyote formaron otra agrupación. Las demás agrupaciones presentaron mezcla entre localidades (Fig. 4).
La composición de macroinvertebrados presentó diferencias significativas respecto a la localidad (ANOSIM, $\mathrm{R}=0.36, \mathrm{p}=$ 0.02). Sámara-Carrillo fue la única localidad que presentó diferencias en la comunidad de macroinvertebrados al comparar con otras localidades individualmente $(\mathrm{R}>0.5, \mathrm{p}<$ $0.05)$ con excepción de Punta Gorda $(\mathrm{R}=1, \mathrm{p}$ $=0.14$ ). De acuerdo al SIMPER, la localidad con mayor similitud entre sitios fue Coyote (66\%), seguida de Sámara-Carrillo (65\%), el Golfo de Papagayo (38\%), San Juanillo (36 $\%)$ y por último Islas Tortuga (31\%). En el cuadro 3 , se pueden observar los porcentajes de disimilitud entre las localidades y las tres especies más importantes que contribuyeron a esas diferencias. 


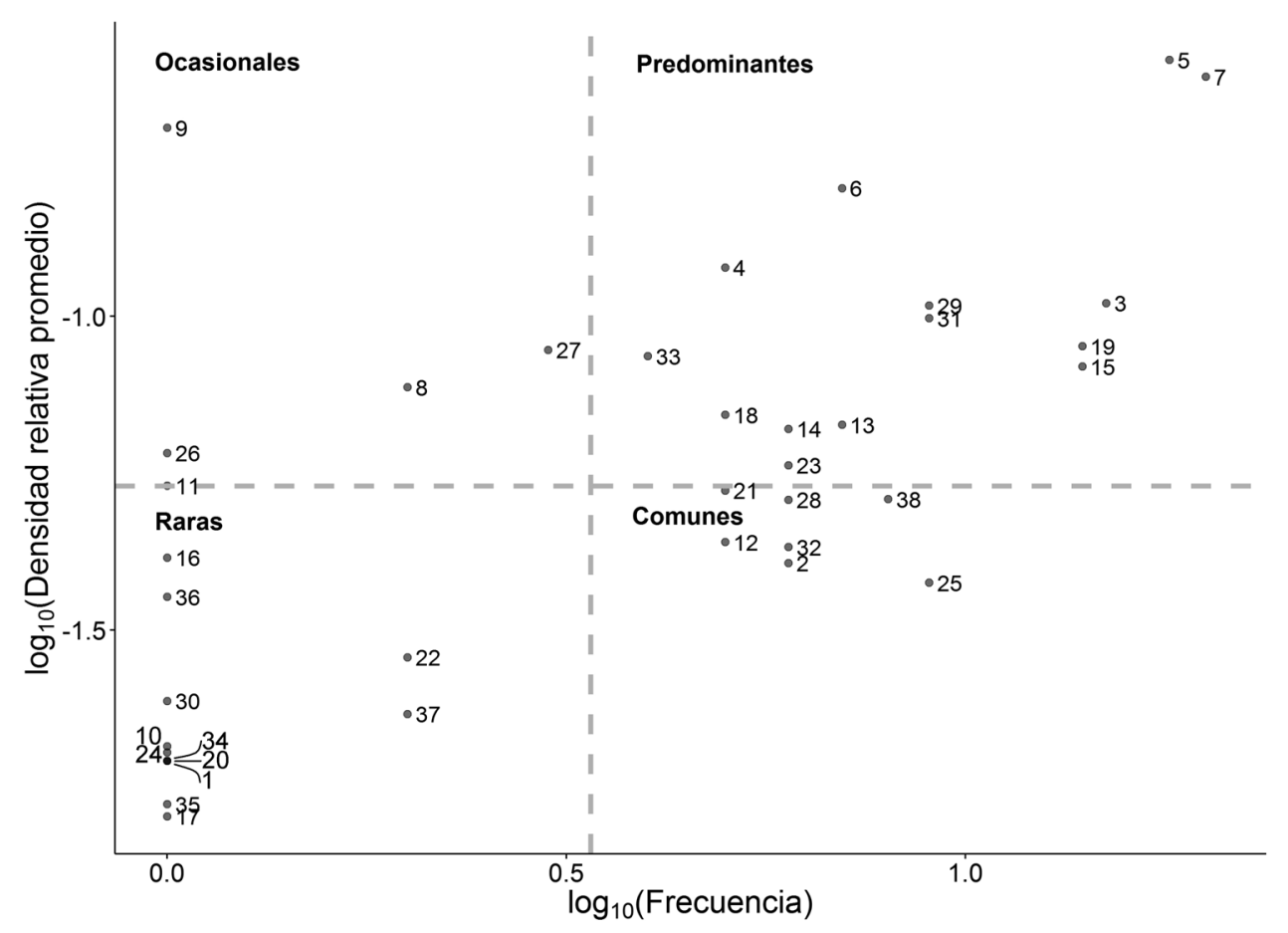

Fig. 3. Análisis de dominancia específica de macroinvertebrados arrecifales en el Pacífico Norte de Costa Rica. Las categorías de dominancia se basaron en la ocurrencia específica y la densidad relativa promedio. Las líneas grises indican el valor promedio de ambas variables. Los números de cada especie se pueden ver en el Cuadro 1.

Fig. 3. Analysis of specific dominance of reef macroinvertebrates in the North Pacific of Costa Rica. The dominance categories were based on the specific occurrence and the average relative density. The gray lines indicate the average value of both variables. The numbers of each species can be seen in Table 1 .

Peces arrecifales: A lo largo de la zona de estudio se contabilizaron 21043 individuos de peces arrecifales, en 94 especies pertenecientes a 37 familias (Anexo 2). El tiburón nodriza (Ginglymostoma unami) fue la única especie de pez cartilaginoso encontrada en la zona, siendo los demás peces óseos (Cuadro 4). Veinticinco especies son frecuentes y con alta abundancia en los sitios de muestreo, entre los que resaltan varias especies de importancia comercial, como el pargo manchado Lutjanus guttatus y el pargo cola amarilla Lutjanus argentriventris. Especies planctívoras como Chromis atrilobata y Abudefduf troschelii fueron también predominantes. Diecisiete especies fueron comunes, mientras que el resto fueron ocasionales o raras (Fig. 5).
En promedio ( \pm error estándar), los arrecifes del Pacífico Norte tienen $1.5( \pm 0.28) \mathrm{t}$ $\mathrm{ha}^{-1}$ de peces. A pesar de que se puede observar un traslape entre las localidades (Fig. 6), estas tienen un efecto en la agrupación de los sitios de acuerdo a la composición de la biomasa de las especies (ANOSIM, $\mathrm{R}=0.35, \mathrm{p}=0.001$ ). Según el análisis de similitud porcentual (SIMPER), los sitios de la localidad Golfo de Papagayo son los que menos se parecen entre sí, con un $15 \%$ mientras que las Islas Murciélago, Sámara Carrillo, Coyote e Islas Tortuga tienen porcentajes de similitud que van entre los 21 \% y $29 \%$. San Juanillo fue la localidad que más difirió con respecto a las Islas Murciélago y el Golfo de Papagayo (Cuadro 3). El Cuadro 3 muestra cuales son las principales especies 
CUADRO 2

Riqueza específica (S), índice de Heterogeneidad de Shannon $\left(H^{\prime}\left(\log _{10}\right)\right)$ e índice de diferenciación taxonómica $\left(\Delta^{*}\right)$ de macroinvertebrados móviles y peces arrecifales por sitio en el Pacífico Norte de Costa Rica, durante el periodo 2013-2014. El código de sitio puede consultarse en la Figura 1

TABLE 2

Specific richness (S), Shannon Heterogeneity index $\left(\mathrm{H}^{\prime}\left(\log _{10}\right)\right)$ and taxonomic distinctiveness index $(\Delta *)$ of movile macroinvertebrates and reef fishes per site in the North Pacific of Costa Rica, during the 2013-2014 period. The site code can be found in Figure 1

\begin{tabular}{|c|c|c|c|c|c|c|c|}
\hline \multirow{2}{*}{ No. } & \multirow{2}{*}{ Sitio } & \multicolumn{3}{|c|}{ Macroinvertebrados móviles } & \multicolumn{3}{|c|}{ Peces arrecifales } \\
\hline & & $\mathrm{S}$ & $\mathrm{H}^{\prime}\left(\log _{10}\right)$ & $\Delta^{*}$ & $\mathrm{~S}$ & $\mathrm{H}^{\prime}\left(\log _{10}\right)$ & $\Delta^{*}$ \\
\hline 11 & Isla San Pedrito & 7 & 0.21 & 77.74 & 18 & 1.02 & 58.35 \\
\hline 12 & Isla Catalina & 9 & 0.17 & 80.72 & 32 & 1.24 & 55.34 \\
\hline 13 & Isla Las Golondrinas & 8 & 0.33 & 77.00 & 21 & 0.85 & 61.48 \\
\hline 14 & Bajo Pochotes & 9 & 0.15 & 73.30 & 19 & 0.97 & 56.30 \\
\hline 15 & Bajo La Vita & 13 & 0.16 & 80.40 & 35 & 1.21 & 60.13 \\
\hline 16 & Isla Pelada & 12 & 0.46 & 74.98 & 14 & 0.92 & 55.75 \\
\hline 17 & Isla Cocinera 1 & 11 & 0.27 & 84.10 & 14 & 0.68 & 56.33 \\
\hline 18 & Isla Cocinera 2 & 12 & 0.25 & 77.37 & 23 & 0.96 & 58.63 \\
\hline 20 & Bajo Negro & 6 & 0.27 & 85.95 & 23 & 0.89 & 48.90 \\
\hline 21 & Palmitas & 10 & 0.81 & 81.48 & 19 & 0.65 & 59.23 \\
\hline 23 & Güiri-Güiri & 11 & 0.49 & 84.83 & 32 & 0.95 & 59.64 \\
\hline 25 & Playa Blanca & 5 & 0.21 & 87.25 & 11 & 0.88 & 61.67 \\
\hline 26 & Jicaral & 6 & 0.65 & 89.49 & 23 & 1.14 & 56.14 \\
\hline 30 & Esmeralda & 3 & 0.41 & 100.00 & 13 & 0.78 & 61.59 \\
\hline 33 & Pelonas & 8 & 0.72 & 89.19 & 18 & 1.01 & 59.00 \\
\hline 37 & Matapalo & 5 & 0.66 & 90.44 & 20 & 1.06 & 58.29 \\
\hline 81 & Punta Pleito & 8 & 0.90 & 86.59 & 9 & 0.77 & 53.16 \\
\hline 82 & El Santuario & 2 & 0.29 & 83.33 & 26 & 0.61 & 45.08 \\
\hline 98 & Bajo Los Castillo & 11 & 0.85 & 89.68 & 25 & 1.14 & 52.93 \\
\hline 99 & E1 Muñeco & 15 & 0.91 & 90.25 & 24 & 0.85 & 49.02 \\
\hline 100 & Isla Chora 1 & 16 & 1.05 & 89.12 & 15 & 0.93 & 55.94 \\
\hline 101 & Isla Chora 2 & 12 & 0.83 & 83.31 & 25 & 1.09 & 51.93 \\
\hline 102 & Punta Carrillo & 18 & 1.01 & 89.54 & 21 & 0.89 & 58.54 \\
\hline 103 & Punta El Indio & 13 & 1.01 & 89.29 & 8 & 0.76 & 49.84 \\
\hline 104 & El Cambute & 12 & 0.99 & 89.75 & 25 & 0.94 & 51.12 \\
\hline 106 & Punta Islita & 6 & 0.65 & 84.27 & 8 & 0.63 & 49.92 \\
\hline 121 & Bahía Curú & 4 & 0.57 & 92.50 & 17 & 0.60 & 36.12 \\
\hline 122 & El Cirial & 5 & 0.65 & 80.59 & 25 & 1.14 & 54.70 \\
\hline 123 & El Mortero & 17 & 0.90 & 91.55 & 21 & 1.09 & 48.88 \\
\hline 124 & El Reloj & 12 & 0.89 & 90.17 & 26 & 0.73 & 39.18 \\
\hline 125 & Tiburón & 7 & 0.79 & 94.92 & 34 & 1.21 & 54.00 \\
\hline
\end{tabular}

que contribuyen a la disimilitud entre los sitios según la localidad. El pargo manchado, es más abundante en Coyote que en la mayoría de los demás sitios, y por tanto fue la especie que contribuyó más en separar a esa localidad del resto (Cuadro 3). Los peces loros, chanchos (Sufflamen verres) y jureles (Caranx caballus) fueron las especies que más contribuyeron a diferenciar las Islas Murciélago de las otras localidades (Cuadro 3).

\section{DISCUSIÓN}

Cobertura del fondo arrecifal: En el Pacífico Norte de Costa Rica, las evaluaciones arrecifales han estado enfocadas mayormente 
Nivel de estrés $=0.16$

$\%$ de similitud

$-50$

Localidad

- Golfo de Papagayo

- Punta Gorda

- San Juanillo

- Sámara-Carrillo

- Coyote

+ Islas Tortuga

30

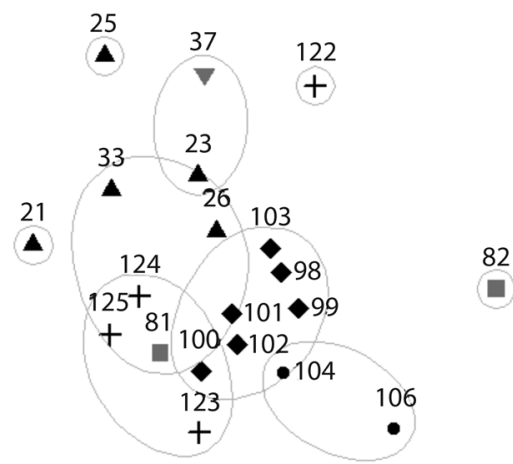

121

$+$

Fig. 4. Análisis multidimensional escalado basado en una matriz de similitud de Bray-Curtis utilizando la densidad de macroinvertebrados del Pacífico Norte de Costa Rica. Las etiquetas identifican las localidades. El código de los sitios puede consultarse en la figura 1.

Fig. 4. Multidimensional scaling analysis based on a Bray-Curtis similarity matrix using the macroinvertebrate density of the North Pacific of Costa Rica. The labels identify the locations. The code of the sites can be consulted in figure 1.

\section{CUADRO 3}

Análisis de similitud porcentual (SIMPER) utilizando densidad de macroinvertebrados (ind $\mathrm{m}^{-2}$ ) y biomasa de peces de acuerdo a la localidad como factor en las localidades de muestreo en el Pacífico Norte de Costa Rica. Se muestra el porcentaje de disimilitud y entre paréntesis las especies que contribuyeron a acumular el $50 \%$ de dichas diferencias en orden descendente. $(*)$ El código de localidad puede consultarse en la Figura 1. (**) El código de especie para macroinvertebrados y peces puede consultarse en el Cuadro 1 y Cuadro 3

TABLE 3

Similarity Percent Analysis (SIMPER) using macroinvertebrate density (ind $\mathrm{m}^{-2}$ ) and fish biomass according to the locality as a factor in the sampling locations in the North Pacific of Costa Rica. The percentage of dissimilarity is shown and between parentheses the species that contributed to accumulate $50 \%$ of those differences in descending order. $(*)$

The locality code can be found in Figure 1. (**) The species code for macroinvertebrates and fish can be found in Table 1 and Table 3

\begin{tabular}{|c|c|c|c|c|c|c|c|c|c|c|}
\hline \multirow{9}{*}{ 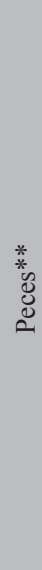 } & & \multicolumn{7}{|c|}{ Localidad* } & & \multirow{9}{*}{ 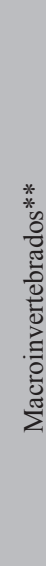 } \\
\hline & & B & $\mathrm{C}$ & D & $\mathrm{J}$ & $\mathrm{L}$ & M & $\mathrm{O}$ & & \\
\hline & B & & - & - & - & - & - & - & B & \\
\hline & $\mathrm{C}$ & $\begin{array}{c}83 \% \\
(74,14,10)\end{array}$ & & $\begin{array}{c}64 \% \\
(27,23,18)\end{array}$ & $\begin{array}{c}67 \% \\
(3,5,29)\end{array}$ & $\begin{array}{c}64 \% \\
(13,28,5)\end{array}$ & $\begin{array}{c}70 \% \\
(5,3,2)\end{array}$ & $\begin{array}{c}68 \% \\
(5,19,7)\end{array}$ & $\mathrm{C}$ & \\
\hline & D & $\begin{array}{c}73 \% \\
(74,14,10)\end{array}$ & $\begin{array}{c}75 \% \\
(72,14,74)\end{array}$ & & $\begin{array}{c}62 \% \\
(3,27,18)\end{array}$ & $\begin{array}{c}67 \% \\
(27,18,13)\end{array}$ & $\begin{array}{c}72 \% \\
(27,3,18)\end{array}$ & $\begin{array}{c}71 \% \\
(27,23,15)\end{array}$ & D & \\
\hline & $\mathrm{J}$ & $\begin{array}{c}93 \% \\
(74,10,14)\end{array}$ & $\begin{array}{c}93 \% \\
(73,14,10)\end{array}$ & $\begin{array}{c}81 \% \\
(72,74,73)\end{array}$ & & $\begin{array}{c}61 \% \\
(13,5,3)\end{array}$ & $\begin{array}{c}48 \% \\
(2,14,19)\end{array}$ & $\begin{array}{c}69 \% \\
(3,15,5)\end{array}$ & $\mathrm{J}$ & \\
\hline & $\mathrm{L}$ & $\begin{array}{c}86 \% \\
(74,10,14)\end{array}$ & $\begin{array}{c}85 \% \\
(36,14,35)\end{array}$ & $\begin{array}{c}79 \% \\
(72,74,68)\end{array}$ & $\begin{array}{c}83 \% \\
(68,36,73)\end{array}$ & & $\begin{array}{c}55 \% \\
(13,5,4)\end{array}$ & $\begin{array}{c}59 \% \\
(13,28,6)\end{array}$ & $\mathrm{L}$ & \\
\hline & M & $\begin{array}{c}91 \% \\
(74,10,54)\end{array}$ & $\begin{array}{c}90 \% \\
(54,88,14)\end{array}$ & $\begin{array}{c}91 \% \\
(54,72,88)\end{array}$ & $\begin{array}{c}73 \% \\
(54,88,91)\end{array}$ & $\begin{array}{c}85 \% \\
(54,88,36)\end{array}$ & & $\begin{array}{c}69 \% \\
(3,2,15)\end{array}$ & M & \\
\hline & $\mathrm{O}$ & $\begin{array}{c}82 \% \\
(35,74,53)\end{array}$ & $\begin{array}{c}84 \% \\
(35,36,54)\end{array}$ & $\begin{array}{c}78 \% \\
(36,35,54)\end{array}$ & $\begin{array}{c}89 \% \\
(36,35,54)\end{array}$ & $\begin{array}{c}76 \% \\
(36,35,53)\end{array}$ & $\begin{array}{c}83 \% \\
(54,36,53)\end{array}$ & & $\mathrm{O}$ & \\
\hline
\end{tabular}




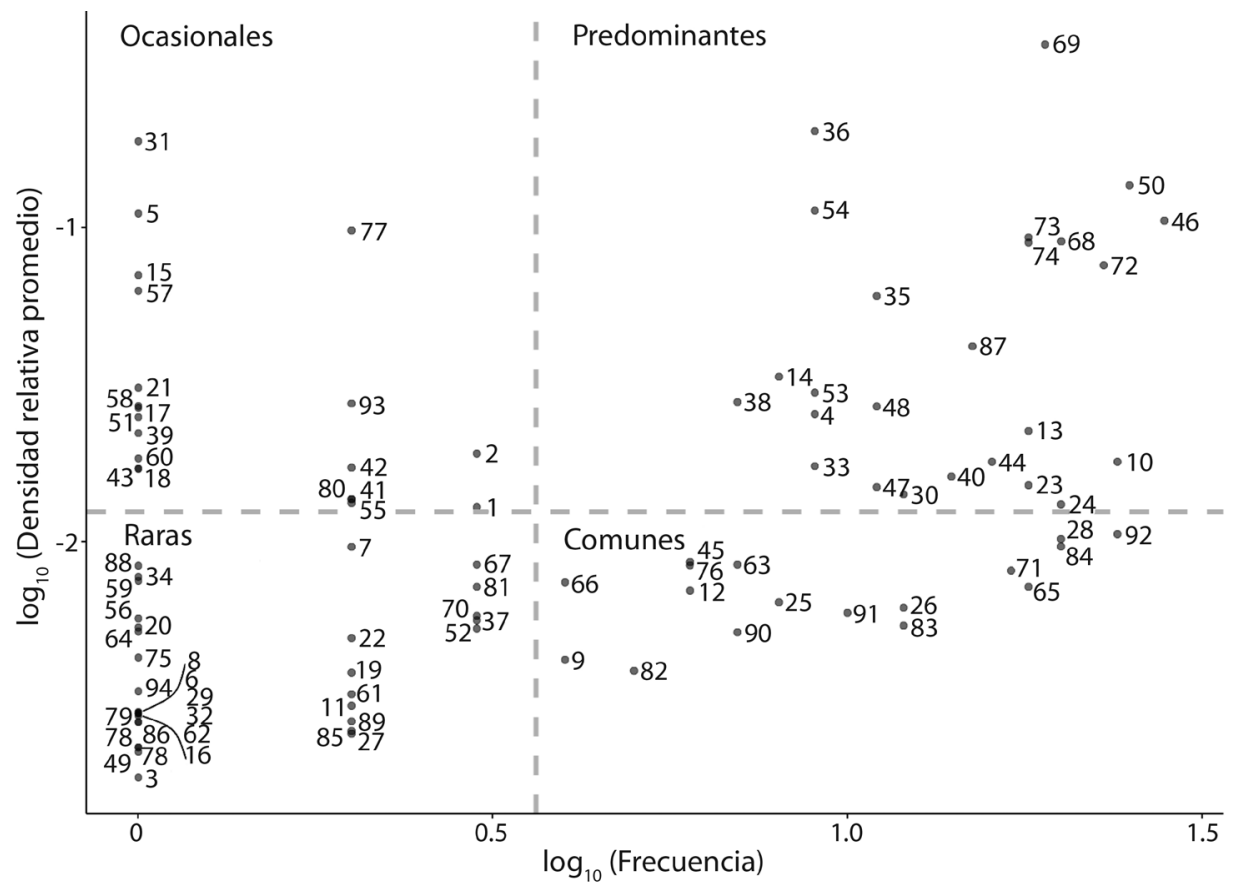

Fig. 5. Análisis de dominancia específica de peces arrecifales en el Pacífico Norte de Costa Rica. Las categorías de dominancia se basaron en la ocurrencia específica y la densidad relativa promedio. Las líneas grises indican el valor promedio de ambas variables. Los números de cada especie se pueden ver en el Cuadro 4.

Fig. 5. Analysis of specific dominance of reef fishes in the North Pacific of Costa Rica. The dominance categories were based on the specific occurrence and the average relative density. The gray lines indicate the average value of both variables. The numbers of each species can be seen in Table 4.
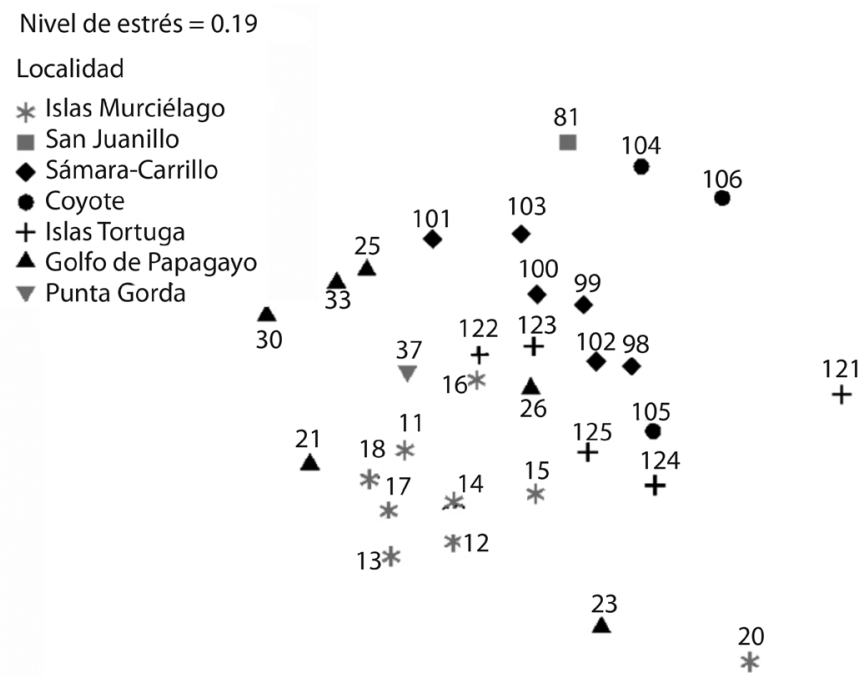

Fig. 6. Análisis multidimensional escalado basado en una matriz de similitud de Bray-Curtis utilizando la biomasa de peces arrecifales del Pacífico Norte de Costa Rica. Las etiquetas identifican las localidades. El código para los sitios puede consultarse en la figura 1.

Fig. 6. Multidimensional scaling analysis based on a Bray-Curtis similarity matrix using the reef fishes biomass of the North Pacific of Costa Rica. The labels identify the locations. The code for the sites can be seen in Figure 1 . 
CUADRO 4

Familias y especies identificadas en los censos de peces de arrecife en el Pacífico Norte.

El número identifica cada especie para análisis posteriores

TABLE 4

Families and species identified in the reef fish census in the North Pacific.

The number identifies each species for later analysis

\begin{tabular}{|c|c|}
\hline & Especie \\
\hline & FAMILIA Acanthuridae \\
\hline 1 & Acanthurus xanthopterus \\
\hline 2 & Prionurus laticlavius \\
\hline 3 & Prionurus punctatus \\
\hline & FAMILIA Apogonidae \\
\hline 4 & Apogon dovii \\
\hline 5 & Apogon retrosella \\
\hline & FAMILIA Aulostomidae \\
\hline 6 & Aulostomus chinensis \\
\hline & FAMILIA Balistidae \\
\hline 7 & Balistes polylepis \\
\hline 8 & Canthidermis maculata \\
\hline 9 & Pseudobalistes naufragium \\
\hline 10 & Sufflamen verres \\
\hline & FAMILIA Belonidae \\
\hline 11 & Belonidae \\
\hline & FAMILIA Blenniidae \\
\hline 12 & Ophioblennius steindachneri \\
\hline 13 & Plagiotremus azaleus \\
\hline & FAMILIA Carangidae \\
\hline 14 & Caranx caballus \\
\hline 15 & Caranx caninus \\
\hline 16 & Caranx sexfasciatus \\
\hline 17 & Elagatis bipinnulata \\
\hline 18 & Gnathanodon speciosus \\
\hline 19 & Seriola rivoliana \\
\hline 20 & Trachinotus rhodopus \\
\hline 21 & Uraspis helvola \\
\hline & FAMILIA Chaenopsidae \\
\hline 22 & Acanthemblemaria hancocki \\
\hline & FAMILIA Chaetodontidae \\
\hline 23 & Chaetodon humeralis \\
\hline 24 & Johnrandallia nigrirostris \\
\hline & FAMILIA Cirrhitidae \\
\hline 25 & Cirrhitichthys oxycephalus \\
\hline 26 & Cirrhitus rivulatus \\
\hline & FAMILIA Diodontidae \\
\hline 27 & Chilomycterus reticulatus \\
\hline 28 & Diodon holocanthus \\
\hline 29 & Diodon hystrix \\
\hline
\end{tabular}

\begin{tabular}{|c|c|}
\hline & Especie \\
\hline \multirow{3}{*}{30} & FAMILIA Fistulariidae \\
\hline & Fistularia commersonii \\
\hline & FAMILIA Gerreidae \\
\hline \multirow[t]{2}{*}{31} & Eucimostomus dowii \\
\hline & FAMILIA Ginglymostomatidae \\
\hline \multirow[t]{2}{*}{32} & Ginglymostoma unami \\
\hline & FAMILIA Haemulidae \\
\hline 33 & Anisotremus caesius \\
\hline 34 & Anisotremus taeniatus \\
\hline 35 & Haemulon flaviguttatum \\
\hline 36 & Haemulon maculicauda \\
\hline 37 & Haemulon sexfasciatum \\
\hline \multirow[t]{2}{*}{38} & Haemulon steindachneri \\
\hline & FAMILIA Holocentridae \\
\hline 39 & Myripristis leiognathus \\
\hline \multirow[t]{2}{*}{40} & Sargocentron suborbitalis \\
\hline & FAMILIA Kyphosidae \\
\hline 41 & Kiphosus sp. \\
\hline 42 & Kyphosus analogus \\
\hline \multirow[t]{2}{*}{43} & Kyphosus elegans \\
\hline & FAMILIA Labridae \\
\hline 44 & Bodianus diplotaenia \\
\hline 45 & Halichoeres chierchiae \\
\hline 46 & Halichoeres dispilus \\
\hline 47 & Halichoeres nicholsi \\
\hline 48 & Halichoeres notospilus \\
\hline 49 & Novaculichthys taeniourus \\
\hline \multirow[t]{2}{*}{50} & Thalassoma lucasanum \\
\hline & FAMILIA Labrisomidae \\
\hline \multirow[t]{2}{*}{51} & Malacoctenus zonifer \\
\hline & FAMILIA Lutjanidae \\
\hline 52 & Hoplopagrus guentherii \\
\hline 53 & Lutjanus argentiventris \\
\hline 54 & Lutjanus guttatus \\
\hline 55 & Lutjanus inermis \\
\hline \multirow[t]{2}{*}{56} & Lutjanus novemfasciatus \\
\hline & FAMILIA Malacanthidae \\
\hline \multirow[t]{2}{*}{57} & Malacanthus brevirostris \\
\hline & FAMILIA Mugilidae \\
\hline 58 & Mugil curema \\
\hline
\end{tabular}


CUADRO 4 (Continuación) / TABLE 4 (Continued)

\begin{aligned} & \multicolumn{1}{c}{ Especie } \\ 59 & Mulloidichthys dentatus \\ 60 & Pseudupeneus grandisquamis \\ & FAMILIA Muraenidae \\ 61 & Gymnothorax castaneus \\ 62 & Muraena argus \\ 63 & Muraena lentiginosa \\ & FAMILIA Phyraenidae \\ 64 & Sphyraena qenie \\ & FAMILIA Pomacanthidae \\ 65 & Holacanthus passer \\ 66 & Pomacanthus zonipectus \\ & FAMILIA Pomacentridae \\ 67 & Abudefduf concolor \\ 68 & Abudefduf troschelii \\ 69 & Chromis atrilobata \\ 70 & Microspathodon bairdii \\ 71 & Microspathodon dorsalis \\ 72 & Stegastes acapulcoensis \\ 73 & Stegastes flavilatus \\ 76 & FAMILIA Scaridae \\ 75 & Scarus ghobban \\ 77 & Scarus rubroviolaceus \\ 78 & Pareques sp. \\ \hline & \end{aligned}

en las zonas de Bahía Culebra, Islas Murciélago y Curú, mientras que para otras zonas este tipo de investigaciones han sido escasas e inclusive más cualitativas que cuantitativas. En estas otras localidades, como Sámara o Cabo Blanco, se indica la presencia de parches arrecifales formados principalmente por Pocillopora spp. o colonias aisladas de Porites lobata, pero no se brinda mayor información de cobertura de coral vivo o de la extensión del arrecife (Cortés, \& Jiménez, 2003).

En las Islas Murciélago se reporta, entre 1994 y 1995, una cobertura promedio de coral de entre 4.8 y $80.9 \%$, con parches arrecifales de hasta $2000 \mathrm{~m}^{2}$ construidos principalmente por Pocillopora spp. (Cortés, 1996-1997b). En Curú, para el 2003, se indica la presencia de un arrecife construido predominantemente

\begin{aligned} & \multicolumn{1}{c}{ Especie } \\ 79 & EAMILIA Scombridae \\ & FAMILIA Scorpaenidae \\ 80 & Scorpaena mystes \\ & FAMILIA Serranidae \\ 81 & Alphestes immaculatus \\ 82 & Alphestes multiguttatus \\ 83 & Cephalopholis panamensis \\ 84 & Epinephelus labriformis \\ 85 & Paranthias colonus \\ 86 & Rypticus nigripinnis \\ 87 & Serranus psittacinus \\ & FAMILIA Sparidae \\ 88 & Calamus brachysomus \\ & FAMILIA Synodonthidae \\ 89 & Synodus lacertinus \\ & FAMILIA Tetraodontidae \\ 90 & Arothron hispidus \\ 91 & Arothron meleagris \\ 92 & Canthigaster punctatissima \\ 93 & Sphoeroides lobatus \\ & FAMILIA Zanclidae \\ 94 & Zanclus cornutus \\ \hline & \end{aligned}

por Psammocora spp. con una cobertura de coral vivo promedio de $49 \%$ y una extensión de 0.24 ha (Bezy et al. 2006). En la actualidad estos arrecifes están muertos y cubiertos por capas de turf.

En Bahía Culebra, la cobertura promedio de coral vivo entre 1995 y 1996 , era de 43.9 $\%$ en arrecifes coralinos (AC), $19.5 \%$ en comunidades coralinas sobre basalto (CCB) y $21.5 \%$ en comunidades coralinas sobre arena (CCA), con coberturas bajas de macroalgas ( $5 \%$ ) (Jiménez, 2001a). Para ese periodo, se reporta $10 \%$ de turf en $\mathrm{AC}$, mientras que en CCB y CCA su cobertura estuvo cercana al 60 \% (Jiménez, 2001a). Posteriormente, Jiménez (2007) reporta degradación extrema en el arrecife de Güiri (Viradores) entre 1990 y 2006, con una disminución en la cobertura promedio 
de coral vivo de $80 \%$ a tan solo $10 \%$; la cual, se atribuye a la ocurrencia de diferentes perturbaciones ambientales en periodo cortos de tiempo, lo cual significó una baja oportunidad de recuperación entre eventos. De acuerdo a Jiménez (2007), los principales disparadores del deterioro coralino han sido el fenómeno de El Niño (1987, 1997, 2003 y 2007), escombros de construcción y FAN (2003, 2004 y 2007). Entre 2010 y 2012, Sánchez-Noguera (2012) reevalúa cuatro de los nueve arrecifes coralinos descritos por Jiménez (2001a), y concluye que dichos ecosistemas sufrieron una degradación extrema en el periodo de 15 años. SánchezNoguera reporta coberturas promedio de coral vivo que oscilan entre 1 y $4 \%$, acompañadas por un aumento significativo en la cobertura de macroalgas. Esta mortandad coralina deja espacio disponible para el asentamiento de otras especies invasoras de rápido crecimiento como el alga invasora $C$. sertularioides (Fernández, \& Cortés, 2005; Fernández-García, 2007; Fernández-García et al., 2012), que modificó por completo el sistema, inclusive transformándolo en un nuevo ecosistema (praderas de Cauler$p a)$. A su vez, la alta disponibilidad de otras algas favoreció el desarrollo de organismos herbívoros, que rápidamente por su acción de alimentación fueron bioerosionando el basamento coralino (Alvarado et al., 2012a, 2016a). Este es el caso del erizo de mar D. mexicanum, cuyas poblaciones previas al 2000 fueron inferiores a 1 ind $\mathrm{m}^{-2}$, que luego de periodos con FAN sus poblaciones aumentaron hasta 8 ind $\mathrm{m}^{-2}$, lo que implicó una actividad bioerosiva intensa (Alvarado et al., 2012a, 2016a).

A partir del 2000, el Pacífico Norte de Costa Rica experimenta un acelerado proceso de alteración costera debido al desarrollo no planificado (campos de golf, actividades agrícolas y aguas residuales), generando mayor cantidad de fuentes de nitrógeno antropogénico (Fernadez-García, 2007; Sánchez-Noguera, 2012). El incremento espacio-temporal de las FAN en la región (Morales-Ramírez et al., 2001; Vargas-Montero et al., 2008) coincidió a su vez con esta tendencia en la alteración costera (Sánchez-Noguera, 2012). La presencia de dinoflagelados en altas densidades suele implicar mayor demanda en el consumo de oxígeno, a la vez, limita la penetración de la radiación solar, provocando estrés a los corales y la consecuente modificación de la estructura arrecifal (Lapointe, Barile, Littler, \& Littler, 2005; Bauman, Burt, Feary, Marquis, \& Usseglio, 2010). La reducción aguda y crónica de la cobertura coralina puede estar asociada al deterioro en la calidad del agua por sedimentos, nutrientes, o enfermedades, lo que provoca una importante reducción en el reclutamiento coralino y en la acreción arrecifal (Flower et al., 2017). Sin embargo, para la zona no existen evidencia que la sedimentación o la presencia de enfermedades sean factor perjudicial para los arrecifes (Jiménez et al., 2010). Esto deja al enriquecimiento de nutrientes como uno de los factores más probables para entender el deterioro de los ecosistemas arrecifales de estas localidades. Por lo tanto, se reafirma en el hecho que un incremento de nutrientes explica un aumento en la cobertura de macroalgas y turf (Férnandez-García et al., 2012; Flower et al., 2017). Por su parte, el turf reduce o previene el asentamiento coralino vía actividad microbiana, lo que afecta negativamente la sobrevivencia de corales (Smith et al., 2016), lo que provoca que el deterioro de los arrecifes del Pacífico Norte sea cíclico, donde el aumento de nutrientes lleva a una mortandad coralina, favoreciendo a sus competidores (turf), que a su vez inhiben el reclutamiento coralino. De manera similar, en las islas del Pacífico central, se determinó que el aumento en la predominancia de turfs y la disminución intensa de corales fue resultado de eutroficación por actividad humana (Jouffray et al., 2014; Smith et al., 2016). En el Pacífico Norte de Costa Rica, las altas coberturas de turf son indicador de la sobrepesca y el enriquecimiento de nutrientes que sufre la zona (Roth, Stuhldreier, SánchezNoguera, Morales-Ramírez, \& Wild, 2015; Stuhldreier et al., 2015a,b,c).

Macroinvertebrados móviles: Los esfuerzos de investigación biológica en el Pacífico Norte de Costa Rica se han enfocado 
principalmente en los arrecifes coralinos, comunidades coralinas y zooplancton (Cortés, 2012, 2017). Muy pocos estudios han sido dirigidos a interpretar aspectos ecológicos de macroinvertebrados en la zona, a excepción de algunos que se enfocaron en especies particulares como Lobatus galeatus (Arroyo-Mora, 1998), A. pulvinata (Alvarado, 2008) y P. gracilis (Naranjo-Madrigal, 2011).

Los invertebrados marinos proveen de importantes servicios ecosistémicos, como lo son el filtrado y purificación del agua, procesamiento de materia orgánica, creación de hábitats, reciclaje de nutrientes y transferencia de carbono en las cadenas tróficas (Collier, Probert, \& Jeffries, 2016). A su vez, estos organismos proveen de varios bienes y servicios para los humanos incluyendo recursos alimenticios claves. De manera general, se ha cuantificado que una alta diversidad de invertebrados es un reflejo de la diversidad y disponibilidad de hábitats (Collier et al., 2016; Hermosillo-Nuñez, Rodrígez-Zaragoza, Ortiz, Calderón-Aguilera, \& Cupul-Magaña, 2016). Sin embargo, cinco agentes son los principales causantes de su disminución: 1) contaminación del agua, 2) sobreexplotación del recurso, 3) degradación del hábitat, 4) especies invasoras y 5) cambio climático (Collier et al., 2016).

A inicios de la década de 1990, Cortés (1996-1997b) recomendó iniciar investigaciones con organismos de interés comercial en la zona de Guanacaste, justificado en la falta de regulación en las extracciones y en la carencia de argumentos científicos de respaldo. A pesar de esto, hasta la fecha se han realizado pocas investigaciones enfocadas en especies pesqueras en la zona. En otras regiones del mundo, se han observado cambios importantes en la dinámica poblacional de algunas especies debido a cambios ambientales globales, ejemplo de esto son las poblaciones de cefalópodos utilizadas en las pesquerías, que han tenido un incremento en los últimos años (Doubleday et al., 2016). El registro de este tipo de eventos es necesario para mejorar las políticas pesqueras y asegurar los recursos para generaciones futuras.
En la presente investigación, predominaron 14 taxa de macroinvertebrados, destacando por sus abundancias D. mexicanum y E. thouarsii (Fig. 3; Cuadro 1). En estudios realizados en otras regiones del Pacífico Centroamericano, señalan a $D$. mexicanum como especie predominante (Alvarado et al., 2011, 2015, 2016b; Alvarado, Guzman, \& Breddy, 2012). Los erizos de mar son especies claves en los arrecifes y su monitoreo es fundamental para poder realizar diagnóstico de la salud de los arrecifes y comunidades coralinas. Un incremento en la población de D. mexicanum disminuye la cobertura algal y puede provocar una fuerte bioerosión (Flower et al., 2017). El aumento en las poblaciones de Diadema puede deberse a la reducción de sus depredadores y el incremento de nutrientes, lo cual favorece el reclutamiento de juveniles que causan mayor pastoreo y fuerte actividad bioerosiva (Flower et al., 2017).

Especies de interés comercial como el Cambute Rey (L. galeatus), los pepinos de mar o las langostas han tenido muy bajo nivel de protección en la región (Arroyo-Mora 1998; Alvarado, Barraza, \& Sancho-Mejía, 2013a). En el caso del Cambute su extracción, captura y comercialización están prohibidos (Sistema Costarricense de Información Jurídica [SCIJ], 2017), y poblaciones sanas sólo se observan dentro de áreas marinas protegidas con un alto grado de protección (i.e. Reserva Natural Absoluta Cabo Blanco) (Arroyo-Mora, 1998; Sibaja-Cordero, Camacho-García, \& VargasCastillo 2014). La población de cambute rey del Pacífico Norte de Costa Rica requiere atención inmediata por parte de las autoridades gubernamentales. La extracción ilegal y la utilización de artes de pesca no selectivos, como la pesca de arrastre, amenazan con reducir la población de este gasterópodo hasta la extinción.

De acuerdo con la Unión Internacional para Conservación de la Naturaleza (UICN), $1.9 \%$ de las especies de pepinos de mar se encuentra amenazadas, $2.4 \%$ son vulnerable a extinguirse, $29.9 \%$ presenta preocupación menor y $65.8 \%$ tienen datos insuficientes (Collier et al., 2016). En el presente estudio, se 
registraron las especies en categoría amenazada (Isostichopus fuscus) (Mercier et al., 2013), en preocupación menor (Holothuria (Halodeima) atra, Holothuria (Mertensiothuria) hilla y Holothuria (Selenkothuria) lubrica) (Conand, Gamboa, \& Purcell, 2013; Alvarado, Ortiz, Benavides, \& Toral-Granda, 2013) y con datos insuficientes (Holothuria (Thymiosycia) arenicola y Holothuria (Cystipus) casoae) (Conand, \& Gamboa, 2013; Samyn, 2013). En el Pacífico Norte de Costa Rica, la pesca furtiva e ilegal de pepinos de mar es una actividad normal, la cual afecta seriamente las poblaciones y el hábitat donde ellas se encuentran (Alvarado et al., 2013a). Esto se ha visto favorecido por una legislación permisiva de extracción sin fundamentos biológicos o pesqueros, sin una cuota, época reproductiva definida o especie clave.

En el caso de la langosta del pacífico Panulirus gracilis, sus poblaciones en la zona se encuentran al borde del colapso (tasa de explotación $=0.8$ ), ya que bajo el régimen actual de pesca no se respetan las tallas mínimas de captura y se extraen hembras portadoras de huevos (Naranjo-Madrigal, 2011). En el presente estudio, $P$. gracilis fue especie predominante, sin embargo, su densidad relativa promedio se mantuvo cerca del valor central. La mayor densidad de este organismo fue observada en Islas Tortuga y Sámara-Carrillo, con máximo de 0.1 ind $\mathrm{m}^{-2}$, densidad similar a la observada en el Pacífico Sur de Costa Rica (Alvarado et al., 2015) y la Isla del Coco (Alvarado et al., 2016b).

Peces arrecifales: Existen pocos trabajos que estudien las comunidades de peces del Pacífico Norte de Costa Rica, y estos se han enfocado en zonas muy localizadas de esta región (Dominici-Arosemana, Brugnoli-Olivera, Cortés- Núñez, Molina-Ureña, \& Quesada-Alpízar, 2005; Espinoza, \& Salas, 2005). En contraste, esta investigación permite complementar una visión amplia de la composición de los ensamblajes de peces en esta costa del país. Dominici-Arosemena et al. (2005) reportan un total de 70 especies para el Golfo de Papagayo en 1997, mientras que Espinoza,
\& Salas (2005) encontraron 43 especies en las Islas Catalinas en el 2003. Ambos estudios consideraron que la zona estudiada es menos diversa que otras regiones arrecifales como Hawái (129 spp.), el mar rojo (199 spp.) y el Golfo de Chiriquí en Panamá (129 spp.). En el presente trabajo, se cuantificaron 94 especies, lo cual es similar a lo que se ha visto en otras zonas arrecifales del Pacífico costarricense como la zona sur (Alvarado et al., 2015) o la Isla del Coco (Alvarado et al., 2016b), con 90 y 93 especies respectivamente, pero superior a sitios como San Juan del Sur en Nicaragua (Alvarado et al., 2011) que en el 2009 se encontraron 53 especies.

MacNeil et al. (2015), al hacer un análisis de las biomasas de peces de varias regiones del mundo, determinan que un arrecife se encuentra en una condición semejante a la prístina cuando posee biomasa superior a $1 \mathrm{t} \mathrm{ha} \mathrm{ha}^{-1}$. En este sentido, las comunidades del Pacífico Norte de Costa Rica estarían mostrando indicadores de buena salud arrecifal. Sin embargo, la biomasa promedio de $1.5 \mathrm{t} \mathrm{ha}^{-1}$ que presentó el Pacífico Norte es muy inferior a las $12.5 \mathrm{t} \mathrm{ha}^{-1}$ que se han encontrado en zonas mejor conservadas como la Isla del Coco (Fourriere, 2016). Además, este trabajo presenta evidencia de una importante degradación del hábitat, reflejada en la cobertura de coral, así como en la composición de invertebrados. De manera que, aunque la diversidad muestre valores semejantes a otros sitios, la baja biomasa puede deberse a una disminución en el tamaño y la densidad de las especies producto de la pérdida de hábitat y la pesca intensiva característica de la zona (Villalobos-Rojas, Herrera-Correal, GaritaAlvarado, Clarke, \& Beita-Jiménez, 2014). Esto acompañado por una gran cantidad de especies que solo se presentan ocasionalmente (Fig. 5). Por lo tanto, las conclusiones de MacNeil et al. (2015) no parecen ser aplicables a los arrecifes de Costa Rica, y dado que los indicadores de biomasa de peces en arrecifes son una opción sencilla para la toma de decisiones en el manejo de pesquerías en situaciones deficientes en datos (McClanahan, Graham, MacNeil, \& Cinner, 2015), es importante actualizar estos 
valores utilizando modelos globales sin la exclusión de regiones, así como también hacer cálculos específicos para cada región.

La especie C. atrilobata fue la más abundante en el estudio de Espinoza \& Salas (2005), y el presente trabajo también constituye una de las especies predominantes. Este pez, al igual que $A$. troschelii, la cual también fue predominante, son planctivoros (Froese, \& Pauly, 2016). Se ha visto que este grupo trófico es dominante en los arrecifes del Pacífico Tropical Oriental (Espinoza, \& Salas, 2005) y no parecen ser especies muy afectadas por las actividades humanas como la pesca (Beita-Jiménez et al., in prep.). Esto se puede deber a que los planctívoros no suelen ser especies de importancia comercial y su alimento no depende de la complejidad estructural de sistemas como arrecifes. El Pacífico Norte es una zona altamente productiva, bajo la influencia de afloramiento costero estacional (Alfaro et al., 2012; Fernández-García et al., 2012) por lo cual es de entender que las especies planctívoras dominen este sistema.

Los pargos también resultaron predominantes en los sitios estudiados, especialmente en Coyote, ya que fue el pargo manchado el que más contribuyó a las diferencias con otras localidades. El Pacífico Norte es una zona con gran actividad pesquera, la cual se desarrolla de manera artesanal y multiespecífica (Villalobos-Rojas et al., 2014). Sin embargo, el pargo manchado es una de las principales especies objetivo de la región (Villalobos-Rojas et al., 2014). En la localidad de Coyote hay una comunidad pesquera que se dedica a la captura de pargo manchado mediante líneas de mano y palangre de fondo, y a lo largo de varios años han venido haciendo esfuerzos para que su pesquería pueda optar por una certificación de sostenibilidad (Villalobos-Rojas et al., 2014). De manera que las características rocosas de la zona y los esfuerzos por hacer una pesca responsable pueden ser los causantes de que en esa localidad se encuentren mayores densidades de pargos. Muchos de los organismos identificados en este estudio fueron juveniles, por lo cual las densidades no reflejan que son poblaciones saludables que toleran más pesca, sino más bien requieren más atención para determinar si son áreas de crianza importante para la recuperación de poblaciones explotadas.

San Juanillo fue un sitio que presentó alta disimilitud con respecto a las Islas Murciélago y al Golfo de Papagayo. Esta localidad se encuentra también rodeada por un pueblo de pescadores que ejercen una importante presión sobre esos recursos (Villalobos-Rojas et al., 2014); por lo cual, es de esperar que tenga comunidades distintas. Por su parte el Golfo de Papagayo, si bien está afectado por actividades humanas como el turismo y pesca, se encuentra en una zona altamente productiva, lo cual beneficia las comunidades de peces en la zona. Las Islas Murciélago, son parte del área marina protegida más grande de la zona (Fig. 1). Las especies que separan esta localidad de las demás no son las más importantes para la pesca artesanal; sin embargo, los peces "loro" y "chancho" son capturados en la pesca mediante buceo (Villalobos-Rojas et al., 2014), por lo cual la protección podría estar teniendo efecto en aumentar las abundancias de algunas especies y es un factor que merece un estudio más detallado.

\section{Implicaciones de manejo para el Pací-} fico Norte: Los ambientes arrecifales poseen gran capacidad de recuperación, pero esta se ve reducida ante la falta de acciones que permitan la protección oportuna de estos sistemas (Cortés, 2007). Por su parte, los peces arrecifales tienen la capacidad de ser resilientes a cambios en la cobertura de coral vivo, siempre y cuando esta se mantenga por encima de $10-20 \%$. Sin embargo, cambios significativos (>20\%) pueden tener grandes efectos en su abundancia, biomasa, redundancia funcional, $\mathrm{y}$ en la productividad pesquera, provocando que estos sistemas sean aún más vulnerables a futuros disturbios (naturales y antropogénicos) (Pratchett, Hoey, \& Wilson, 2014). La educación ambiental y el trabajo de conservación en conjunto con las comunidades costeras son claves para favorecer la recuperación de los ambientes marinos y permitir la utilización 
sustentable de los recursos. La degradación y perdida del hábitat son las principales causas de declive de la biodiversidad, y por lo tanto de sus bienes y servicios. Una perdida en la complejidad del hábitat reduce el número de organismos que en él viven, y de ser continua, como en el caso de los arrecifes coralinos puede llevar a una importante reducción en la abundancia de peces (Pratchett et al., 2014). Los arrecifes construidos principalmente por el coral Pocillopora spp. albergan mayor número de especies de invertebrados a nivel mundial; sin embargo, son los que poseen la mayor vulnerabilidad ante disturbios (Stella, Pratchett, Hutchings, \& Jones, 2011). En la presente evaluación hemos encontrado que la cobertura de coral vivo promedio de la costa del Pacífico Norte de Costa Rica es de apenas $5 \%$, lo cual representa pérdida de hasta $100 \%$ en algunas localidades, La condición crítica en la que se observaron los arrecifes de esta región del país, nos hace cuestionarnos ¿Qué impacto tiene, sobre la salud del océano y de los servicios que este brinda a las comunidades costeras, el que desaparezcan los arrecifes coralinos del Pacífico Norte? y, ¿qué estamos haciendo para revertir este proceso? ¿Se están controlando adecuadamente el desarrollo costero y la actividad turística? ¿Por qué no existen los mecanismos para controlar y dar seguimiento a la condición de los ecosistemas marino costeros? ¿Por qué las acciones de manejo de biodiversidad, pesquero, de cuencas y agrícolas, cuando existen, no son efectivas?

El Pacífico Norte de Costa Rica posee condiciones biofísicas ideales para el desarrollo de arrecifes coralinos, debido a una estación lluviosa menos intensa y corta, la ausencia de fuertes escorrentías y fuertes eventos de sedimentación, y a presencia de múltiples bahías pequeñas con condiciones calmas de oleaje (Jiménez et al., 2010). Bajo estas características, la recuperación de los ambientes arrecifales del Pacífico Norte es posible, pero requiere múltiples y grandes esfuerzos paralelos para que el proceso natural de regeneración tenga éxito. Las amenazas que enfrentan nuestros recursos marino costeros deben ser manejadas de forma integral, con un compromiso por parte de las autoridades, acompañado de una fuerte organización comunitaria y el trabajo conjunto con entidades académicas y organizaciones no gubernamentales que permitan implementar medidas efectivas y duraderas para la recuperación y conservación de los ecosistemas marinos. Este compromiso requiere el manejo integral de cuencas, efectivo ordenamiento espacial marino-costero (que incluya reglamentos de uso de los recursos), manejo efectivo de las áreas marinas protegidas, el control sobre las regulaciones pesqueras y ambientales existentes, controlar las entradas de aguas (servidas y negras) no tratadas al mar, realizar y ejecutar estudios de capacidad de carga para la actividad turística, establecer programas de control de las especies invasoras, poner en práctica la jardinería y restauración arrecifal, desarrollar un manejo ecosistémico de las pesquerías, ampliar los programas de educación ambiental marino costeros y continuar con los programas de monitoreo biológico. Ante este panorama, el manejo ecosistémico se vuelve crucial ante la posible entrada de amenazas mayores a los ecosistemas de la región, como lo son la intención de crear un canal interoceánico en Nicaragua (Alvarado et al., 2016b) y la propuesta de desarrollar un canal seco en el Norte de Costa Rica (http://www.elmundo.cr/costa-ricapresenta-proyecto-canal-seco-interoceanico16-billones-dolares/). Esto pondría en riesgo la gran cantidad de recursos marino-costeros en la zona del Parque Nacional Santa Rosa, una de las localidades menos deterioradas del Pacifico Norte. La creación de ambos proyectos podría contribuir con la degradación de los arrecifes coralinos de la región.

El desarrollo costero no debe implicar deterioro ambiental. La salud de los ecosistemas marinos y terrestres está ligada a la salud de las poblaciones humanas que en él habitan, y de las actividades económicas que de estos dependen. La costa del Pacífico Norte de Costa Rica, hace tan sólo 20 años fue considerada como la zona con mayor y mejor desarrollo arrecifal del país, sin embargo, el continuo abuso y sobreexplotación de sus recursos ha 
socavado significativamente su riqueza y en consecuencia los servicios que proveía. Es urgente realizar un cambio en la conciencia ambiental de las autoridades, de las comunidades y de todos los usuarios, la zona depende del turismo el cual a su vez depende de la salud de los ecosistemas marino-costeros. Si la zona se quiere recuperar y mantener como un motor económico para el país, debe tomarse decisiones integrales de manejo urgentes o en su lugar se verá convertida en una bomba de tiempo de deterioro ambiental, social y económico.

Ante las múltiples amenazas (naturales y antropogénicas) que enfrentan los ecosistemas marino costeros a nivel mundial y en nuestro país, el desarrollo de medidas efectivas de manejo y conservación sobre estos recursos es inminente. Para consolidar medidas de gestión efectivas es necesario respaldarse en el conocimiento científico que evalué, valide y realice el monitoreo de la condición de los ecosistemas y su biodiversidad. Sin embargo, en nuestro país existe un vacío importante debido a la ausencia de un sistema efectivo, eficaz y eficiente que genere y analice información científica sobre el estado de las especies y ecosistemas. Sumado a ello, en muchos casos, no se cuenta con una línea base que permita asegurar la permanencia y funcionalidad de los recursos marinos. Al carecer de este seguimiento, sumado al constante aumento de las amenazas como el desarrollo costero no planificado, hace que los sistemas coralinos analizados en este estudio y que caracterizaron a la costa Pacífica Norte de Costa Rica se encuentren ampliamente afectados.

\section{AGRADECIMIENTOS}

La presente investigación no se hubiera podido realizar sin el apoyo en campo de las siguientes personas: N. Sandoval, K. García, J. Quirós, A. Sánchez, M. Méndez, J. S. Vargas, J. Nivia, O. Breedy, O. Esquivel, V. Bogantes, G. Vega, C. Salas, J. C. Azofeifa, A. Terán y Familia, C. Benavides, M. M. Chavarría, L. Shutt y PRETOMA. Asimismo, se agradece el apoyo logístico de los funcionarios y guardarparques del Refugio de Vida Silvestre Curú, Reserva Natural Absoluta Cabo Blanco, Parque Marino Las Baulas, Refugio de Vida Silvestre CaletasAgrio y del Parque Nacional Santa Rosa (sector marino). Se agradece a la Agencia Alemana de Cooperación GiZ a través del proyecto BIOMARC, Asociación Costa Rica por Siempre, Conservación Internacional Costa Rica y la Vicerrectoría de Investigación de la Universidad de Costa Rica (Proyectos 808-98-013, 808A5-037, 808-B3-503) por el apoyo económico. El presente proyecto se encuentra inscrito en FundaciónUCR (2739-019) y en Vicerrectoría de Investigación de la Universidad de Costa Rica (808-98-013, 808-A5-037, 808-B3-503).

\section{RESUMEN}

Los arrecifes de coral son ecositemas muy diversos y productivos, a pesar de eso, están siendo amenazados por actividades humanas que potencian fenómenos naturales nocivos como las Floraciones Algales Nocivas (FAN). El pacífico norte de Costa Rica se ha caracterizado como una de las regiones de mayor desarrollo de arrecifes del país, sin embargo, muchos de estos ecosistemas se están perdiendo producto de la eutrofización, sobrepesca, especies invasoras entre otras que afectan la región. Este trabajo muestra que la cobertura coralina en el pacífico norte es en promedio de $5.0 \pm 10.4$ (d.e) $\%$, pasando a ser arrecifes dominados por turf. Se registraron 26 taxa de macroinvertebrados en la zona, siendo el erizo de mar Diadema mexicanum una especie predominante. Ademas, se identificaron 94 especies de peces arrecifales, de las cuales, las especies de pargos y peces planctívoros fueron los que se presentaron en mayor frecuencia y abundancia. Para las zonas estudiadas en la década de 1990, la cobertura de coral vivo se encontraba entre $40 \%$ y $50 \%$, por lo cual el estado actual refleja deterioro significativo. Esta disminución se debe a eventos naturales como el fenómeno de El Niño, así como por el deterioro de la calidad de agua en la región. Se han reportado en estos años proliferaciones del alga invasiva Caulerpa sertularioides y grandes densidades de erizos de mar bioerosionadores, principalmente asociados a eventos de FAN. De igual forma las comunidades de peces presentan biomasas bajas sobre todo en la cercanía de pueblos pesqueros. El estado de los arrecifes en el pacífico norte es preocupante y requiere acciones para su recuperación y conservación, para lo cual debe haber un mejor planeamiento del desarrollo de proyectos y actividades en la costa.

Palabras clave: Floraciones Algales Nocivas, especies invasoras, desarrollo costero, El Niño, sobrepesca. 


\section{REFERENCIAS}

Alfaro, E. J., Cortés, J., Alvarado, J. J., Jiménez, C., León, A., Sánchez-Noguera, C., Nivia-Ruiz, J., \& Ruiz, E. (2012). Clima y temperatura subsuperfical del mar en Bahía Culebra, Golfo de Papagayo, Costa Rica. Revista de Biología Tropical, 60(Supl. 2), 159-171.

Alvarado, J. J. (2008). Seasonal ocurrence and aggregation behavior of the sea urchin Astropyga pulvinata (Echinodermata: Echinoidea) in Bahía Culebra, Costa Rica. Pacific Science, 62(Supl. 4), 579-592.

Alvarado, J. J., Ayala, A., Álvarez del Castillo-Cárdenas, P. A., Fernández, C., Aguirre-Rubí, J., Buitrago, F., \& Reyes Bonilla, H. (2011). Coral communities of San Juan del Sur, Pacific Nicaragua. Bulletin of Marine Science, 87(Supl. 1), 129-146.

Alvarado, J. J., Cortés, J., \& Reyes-Bonilla, H. (2012a). Bioerosion impact model for the sea urchin Diadema mexicanum on three Costa Rican Pacific coral reefs. Revista de Biología Tropical, 60(Suppl. 2), 121-132.

Alvarado J. J., Guzmán, H. M., \& Breedy, O. (2012). Distribución y diversidad de equinodermos (Asteroidea, Echinoidea, Holothuroidea) en las Islas del Golfo de Chiriquí, Panamá. Revista de Biología Marina y Oceanografia, 47(Supl. 1), 13-22.

Alvarado J. J., Barraza, E., \& Sancho-Mejía, T. (2013a). Chapter 3 Central America Echinoderms: diversity, ecology and future perspectives. En J. J. Alvarado \& F. A. Solís-Marín (Eds.). Echinoderm Research and Diversity in Latin America (pp. 67-106). Berlin, Germany: Springer.

Alvarado, J. J., Ortiz, P., Benavides, M., \& Toral-Granda, T. G. (2013). Holothuria lubrica. The IUCN Red List of Threatened Species 2013: e.T180387A1624294. Recuperado de http://dx.doi.org/10.2305/IUCN. UK.2013-1.RLTS.T180387A1624294.en.

Alvarado, J. J., Beita, A., Mena, S., Fernández-García, C., \& Guzmán-Mora, A. G. (2015). Ecosistemas coralinos del Área de Conservación Osa: análisis estructural y necesidades de conservación. Revista de Biología Tropical, 63(Supl. 1), 219-259.

Alvarado, J. J., Beita-Jiménez, A., Mena, S., Fernández-García, F., Guzmán-Mora, A. G., \& Cortés, J. (2016a). Ecosistemas coralinos del Área de Conservación Marina Isla del Coco, Costa Rica: estructura y comparación 1987-2014. Revista de Biología Tropical, 64(Supl. 1), 153-175

Alvarado, J. J., Aburto-Oropeza, O., Abad, R., Barraza, E., Brandt, M., Cantera, J., Estrada, P., Gaymer, C. F., Guzmán-Mora, A. G., Herlan, J. J., \& Maté, J. L. (2016b) Coral Reef Conservation in the Eastern Tropical Pacific. En P. W. Glynn, D. P. Manzello \& I. C. Enochs (Eds.). Coral Reefs of the Eastern Tropical Pacific (pp. 565-591). Holanda: Springer.
Arroyo-Mora, D. (1998). Crecimiento y reproducción de Strombus galeatus (Gastropoda: Strombidae) en el Pacífico de Costa Rica. Revista Biología Tropical, 46(Supl. 1), 27-36.

Bauman, A. G., Burt, J. A., Feary, D. A., Marquis, E., \& Usseglio, P. (2010). Tropical harmful algal blooms: An emerging threat to coral reef communities? Marine Pollution Bulletin, 60, 2117-2122.

Bezy, M. B., Jiménez, C., Cortés, J., Segura, A., León, A., Alvarado, J. J., Gillén, C., \& Mejía, E. (2006). Contrasting Psammocora-dominated coral communities in Costa Rica, tropical eastern Pacific. Proceedings of the 10th International Coral Reef Symposium (pp. 376-381). Okinawa, Japón: organización simposio o editor.

Birkeland, C. (1997). Life and Death of Coral Reefs. Nueva York: Chapman \& Hall.

Calvo-Vargas, E., Berrocal-Artavia, K., \& Boza-Abarca, J. (2016). Floraciones algales nocivas durante el periodo 2008-2010 en el Golfo de Nicoya, Costa Rica. Revista Ciencias Marinas y Costeras, 8(Supl. 1), 129-149.

Collier, K. J., Probert, P. K., \& Jeffries, M. (2016). Conservation of aquatic invertebrates: concerns, challenges and conundrums. Aquatic Conservation: Marine and Freshwater Ecosystems, 26, 817-837.

Conand, C., \& Gamboa, R. (2013). Holothuria arenicola. The IUCN Red List of Threatened Species 2013: e.T180437A1630561. Recuperado de http://dx.doi.org/10.2305/IUCN.UK.2013-1.RLTS. T180437A1630561.en

Conand, C., Gamboa, R., \& Purcell, S. (2013). Holothuria atra. The IUCN Red List of Threatened Species 2013: e.T180421A1628832. Recuperado de http://dx.doi.org/10.2305/IUCN.UK.2013-1.RLTS. T180421A1628832.en

Conand, C., Purcell, S., \& Gamboa, R. (2013). Holothuria hilla. The IUCN Red List of Threatened Species 2013: e.T180235A1603966. Recuperado de http://dx.doi.org/10.2305/IUCN.UK.2013-1.RLTS. T180235A1603966.en

Cortés, J. (1996-1997a) Biodiversidad marina de Costa Rica: Filo Cnidaria. Revista de Biología Tropical, 44/45(Supls. 3/1), 323-334.

Cortés, J. (1996-1997b) Comunidades coralinas y arrecifes del Área de Conservación Guanacaste, Costa Rica. Revista de Biología Tropical, 44/45(Supls. 3/1), 623-625.

Cortés, J. (1997). Biology and geology of eastern Pacific coral reefs. Coral Reefs, 16(Supl. 1), S39-S46.

Cortés, J. (2007). Arrecifes coralinos de Costa Rica: amenazas y recuperación. Ambientico, 171, 3-4. 
Cortés, J. (2012). Historia de la investigación marinocostera en Bahía Culebra, Pacífico Norte, Guanacaste, Costa Rica. Revista Biología Tropical, 60(Supl. 2), 19-37.

Cortés, J. (2014). Compilación y análisis de las investigaciones científicas sobre temas marinos y atmosféricos en el Pacífico Norte de Costa Rica. Revista Biología Tropical, 62 (Supl. 4), 151-184.

Cortés, J. (2016). Pacific Costa Rica: Coastal and Marine Ecosystems. En M. Kappelle (Ed.). Costa Rican Ecosystems (pp. 73-105). Chicago, EEUU: The University of Chicago Press.

Cortés, J. (2017). Marine biodiversity baseline for Área de Conservación Guanacaste, Costa Rica: published records. ZooKeys, 652, 129-179

Cortés, J., \& Guzmán, H. M. (1998). Organismos de los arrecifes coralinos de Costa Rica: Descripción y distribución geográfica e historia natural de los corales zooxantelados (Anthozoa: Scleractinia) del Pacífico. Revista de Biología Tropical, 46(Supl. 1), 55-91.

Cortés, J., \& Jiménez, C. E. (2003). Corals and coral reefs of the Pacific of Costa Rica: history, research and status. En J. Cortés (Ed.). Latin American Coral Reefs (pp. 361-385). Amsterdam, Netherlands: Elsevier Science B.V.

Cortés, J., Jiménez, C. E., Fonseca A. C., \& Alvarado J. J. (2010). Status and conservation of coral reefs in Costa Rica. Revista de Biología Tropical, 58(Supl 1), 33-50.

Dominici-Arosemena, A., Brugnoli-Olivera, E., CortésNúñez, J., Molina-Ureña, H., \& Quesada-Alpízar, M. (2005). Community structure of eastern Pacific reef fishes (Gulf of Papagayo, Costa Rica). Tecnociencia, 7(Supl. 2), 19-41.

Doubleday, Z. A., Prowse, T. A. A., Arkhipkin, A., Pierce, G. J., Semmens, J., Steer, M., Leporati, S. C., Lourenço, S.,Quetglas, A., Sauer, W., \& Gillanders, B. M. (2016). Global proliferation of cephalopods. Current Biology, 26(Supl. 10), R406-R407.

Espinoza, M., \& Salas, E. (2005). Estructura de las comunidades de peces de arrecifes en las Islas Catalinas y Playa Ocotal, Pacífico Norte de Costa Rica. Revista de Biología Tropical, 53, 523-536.

Fernández-García, C. (2007). Propagación del alga Caulerpa sertularioides (Chlorophyta) en Bahía Culebra, Golfo de Papagayo, Pacífico norte de Costa Rica (Tesis de Maestría). San José, Costa Rica: Universidad de Costa Rica.

Fernández, C., \& Cortés, J. (2005). Caulerpa sertularioides, a green alga spreading aggressively over coral reef communities in Culebra Bay, North Pacific of Costa Rica. Coral Reefs, 24(Supl. 1), 10.
Fernández-García, C., Cortés, J., Alvarado, J. J., \& NiviaRuiz, J. (2012). Physical factors contributing to the benthic dominance of the alga Caulerpa sertularioides (Caulerpaceae, Chlorophyta) in the upwelling Bahía Culebra, north Pacific of Costa Rica. Revista de Biología Tropical, 60(Supl. 2), 93-107.

Flower, J., Ortíz, J. C., Chollet, I., Abdullah, S., CastroSanguino, C., Hock, K., Lam, V., \& Mumby, P. J. (2017). Interpretating coral reef monitoring data: a guide for improved management decisions. Ecological indicators, 72, 848-869.

Fourierre, M. (2016). Energy flow structure and function of keystone groups in shallow water environments in Isla del Coco, Costa Rica, Eastern Tropical Pacific (Tesis de Maestría). Bremen, Alemania: Universidad de Bremen.

Froese, R., \& Pauly, D. (2016). FishBase. World wide web electronic publication. Recuperado de http://www. fishbase.org, version (10/2016)

Glynn, P. W., Druffel, E. M., \& Dunbar, R. B. (1983). A dead Central American coral reef tract: possible link with the Little Ice Age. Journal of Marine Research, 41, 605-637.

Guzmán, H. M., Cortés, J., Glynn, P. W., \& Richmond, R. H. (1990). Coral mortality associated with dynoflagellate blooms in the eastern Pacific (Costa Rica and Panama). Marine Ecology Progress Series, 60, 299-303.

Hermosillo-Nuñez, B. B., Rodríguez-Zaragoza, F. A., Ortiz, M., Calderón-Aguilera, L. E., \& Cupul-Magaña, A. L. (2016). Influence of the coral reef assemblages on the spatial distribution of echinoderms in a gradient of human impacts along the tropical Mexican Pacific. Biodiversity and Conservation, DOI: 10.1007/s10531-016-1182-y

Jiménez, C. (2001a) Arrecifes y ambientes coralinos de Bahía Culebra, Pacífico norte de Costa Rica: aspectos biológicos, económico-recreativos y de manejo. Revista de Biología Tropical, 49(Supl. 2), 215-231.

Jiménez, C. (2001b). Seawater temperature measured at the surface and at two depths (7 and $12 \mathrm{~m})$ in one coral reef at Culebra Bay, Gulf of Papagayo, Costa Rica. Revista de Biología Tropical, 49(Supl. 2), 153-161.

Jiménez, C. (2007). Arrecifes coralinos, ¿víctimas de los cambios? Ambientico 171, 5-7.

Jiménez, C., Cortés, J., León, A., \& Ruiz, E. (2001). Coral bleaching and mortality associated with the 1997-98 El Niño in an upwelling environment in the eastern Pacific (Gulf of Papagayo, Costa Rica). Bulletin of Marine Science, 69, 151-169.

Jiménez, C., Bassey, G., Segura, Á., \& Cortés, J. (2010). Characterization of the coral communities and reefs of two previously undescribed locations in the 
upwelling region of Gulf of Papagayo (Costa Rica). Revista Ciencias Marinas y Costeras, 2, 95-108.

Jouffray, J. B., Nyström, N., Noström, A. V., Williams, I. D., Wedding, L. M., Kittinger, J. N., \& Williams, G. J. (2014). Identifying multiple coral reef regimes and their drivers across the Hawaiian archipelago. Philosophical Transactions of the Royal Society B, 370, 20130268.

Karlson, R. H. (1999). Dynamics of Coral Communities. London: Kluwer Academic Press.

Lapointe, B., Barile, P. J., Littler, M. M., \& Littler, D. (2005). Macroalgal blooms in southeast Florida coral reefs. II. Cross-shelf discrimination of nitrogen sources indicates widespread assimilation of sewage nitrogen. Harmful Algae, 4, 1106-1122.

MacNeil, M. A., Graham, N. A. J., Cinner, J. E., Wilson, S. K., Williams, I. D., Maina, J., \& McClanahan, T. R. (2015). Recovery potential of the world's coral reef fishes. Nature, 520, 341-344.

McClanahan, T.R, Graham, N. A. J., MacNeil, M. A., \& Cinner, J. E. (2015). Biomass-based targets and the management of multispecies coral reef fisheries. Conservation Biology, 29, 409-417.

Mercier, A., Hamel, J. F., Toral-Granda, T. G., Alvarado, J. J., Paola Ortiz, E., \& Benavides, M. (2013). Isostichopus fuscus. The IUCN Red List of Threatened Species 2013: e.T180373A1621878. Recuperado de http://dx.doi.org/10.2305/IUCN.UK.2013-1.RLTS. T180373A1621878.en

Morales-Ramírez, A., Víquez, R., Rodríguez, K., \& Vargas, M. (2001). Marea roja producida por Lingulodinium polyedrum (Peridiniales, Dinophyceae) en Bahía Culebra, Golfo de Papagayo, Costa Rica. Revista de Biología Tropical, 49(Supl. 2), 19-23.

Naranjo-Madrigal, H. (2011). Biología pesquera de la langosta Panulirus gracilis en Playa Lagarto, Guanacaste, Costa Rica. Revista Biología Tropical, 59(Supl. 2), 619-633.

Pratchett, M. S., Hoey, A. S., \& Wilson, S. K. (2014). Reef degradation and the loss of critical ecosystem goods and services provided by coral reef fishes. Current Opinion in Environmental Sustainability, 7, 37-43.

Reaka-Kudla, M. L. (1997). The global biodiversity of coral reefs: A comparison with rainforest. En M. L. Reaka-Kudla, D. E. Wilson \& E.O. Wilson (Eds.). Biodiversity II: Understanding and protecting our natural resources (pp. 83-108.). Washington: Joseph Henry/National Academy Press.

Rixen, T., Jiménez, C., \& Cortés, J. (2012). Impact of upwelling events on the sea water chemistry in the of Gulf of Papagayo (Culebra Bay), Costa Rica. Revista de Biología Tropical, 60(Supl. 2) 187-195.
Roth, F., Stuhldreier, I., Sánchez-Noguera, C., MoralesRamírez, A., \& Wild, C. (2015). Effects of simulated overfishing on the succession of benthic algae and invertebrates in an upwelling-influenced coral reef of Pacific Costa Rica. Journal of Experimental Marine Biology and Ecology, 468, 55-66.

Samyn, Y. (2013). Holothuria casoae. The IUCN Red List of Threatened Species 2013: e.T19708918A19746832. Recuperado de http://dx.doi.org/10.2305/IUCN. UK.2013-1.RLTS.T19708918A19746832.en

Sánchez-Noguera, C. (2012). Cambios socioeconómicos y ambientales en Bahía Culebra, Guanacaste, Costa Rica: implicaciones para su gestión (Tesis de Maestría). San José, Costa Rica: GIACT, Universidad de Costa Rica.

Sistema Costarricense de Información Jurídica. (2017). Sistema Costarricense de Información Jurídica. Recuperado de http://www.pgrweb.go.cr/scij/Busqueda/ Normativa/Normas/nrm_texto_completo.aspx?param $1=$ NRTC\&nValor $1=1 \& \bar{n}$ Valor $\overline{2}=41527 \&$ nValor $3=43$ 776\&strTipM=TC

Sibaja-Cordero, J. A., Camacho-García, Y. E., \& VargasCastillo, R. (2014). Riqueza de especies de invertebrados en playas de arena y costa del Pacífico Norte de Costa Rica. Revista Biología Tropical, 62(Supl. 4), 63-84.

Smith, J. E., Brainard, R., Carter, A., Grillo, S., Edwards, C., Harris, J., Lewis, L., Obura, D., Rohwer, F., Sala, E., Vroom, P. S., \& Sandin, S. (2016). Re-evaluating the health of coral reef communities: baselines and evidence for human impact across the central Pacific. Proceeding of the Royal Society B, 283, 20151985.

Stella, J. S., Pratchett, M. S., Hutchings, P. A., \& Jones, G. P. (2011). Coral-associated invertebrates: diversity, ecological importance and vulnerability to disturbance. Oceanography and Marine Biology: An Annual Review, 49, 43-104.

Stuhldreier, I., Sánchez-Noguera, C., Rixen, T., Cortés, J., Morales-Ramírez, A., \& Wild, C. (2015a). Effects of seasonal upwelling on inorganic and organic matter dynamics in the water column of Eastern Pacific coral reefs. PlosOne, 10, e0142681.

Stuhldreier, I., Sánchez-Noguera, C., Roth, F., Cortés, J., Rixen, T., \& Wild, C. (2015b). Upwelling increases net primary production of corals and reef-wide gross primary production along the Pacific coast of Costa Rica. Frontiers in Marine Science, 2, 113.

Stuhldreier, I., Sánchez-Noguera, C., Roth, F., Jiménez, C., Rixen, T., Cortés, J., \& Wild, C. (2015c). Dynamics in benthic community composition and influencing factors in upwelling-exposed coral reef on the Pacific coast of Costa Rica. PeerJ, 3, e1434.

Vargas-Montero, M., \& Freer, E. (2004). Proliferaciones algales de la diatomea toxigénica Pseudo-Nitzschia 
(Bacillariophyceae) en el Golfo de Nicoya, Costa Rica. Revista de Biología Tropical, 52(Supl. 1), 127-132.

Vargas-Montero, M., Freer-Bustamante, E., Guzmán, J. C., \& Vargas, J. C. (2008). Florecimientos de dinoflagelados nocivos en la costa Pacífica de Costa Rica. Hidrobiológica, 18, 15-23.
Villalobos-Rojas, F., Herrera-Correal, J., Garita-Alvarado, C., Clarke, T., \& Beita-Jiménez, A. (2014). Actividades pesqueras dependientes de la ictiofauna del Pacífico Norte de Costa Rica. Revista de Biología Tropical, 62(Supl. 4), 119-138.

Weinberg, S. (1981). A comparison of coral reef survey methods. Bijdragen tot de Dierkunde, 51, 199-218. 


\section{ANEXO 1}

Densidad promedio (ind $\mathrm{m}^{-2}$ ) y desviación estándar de macroinvertebrados por sitio y localidad en el Pacífico Norte de Costa Rica, 2013-2014. Ver Figura 1 para detalle de los sitios de muestreo)

Average density (ind $\mathrm{m}^{-2}$ ) and standard deviation of macroinvertebrates by site and location in the North Pacific of Costa Rica, 2013-2014. See Figure 1 for detail of the sampling sites)

\begin{tabular}{|c|c|c|c|c|c|c|c|c|c|}
\hline Especie & 11 & 12 & 13 & 14 & 15 & 16 & 17 & 18 & 20 \\
\hline \multicolumn{10}{|l|}{ Filo Echinodermata } \\
\hline \multicolumn{10}{|l|}{ Clase Asteroidea } \\
\hline Mithrodia bradleyi & - & - & - & - & - & - & - & 0.10 & - \\
\hline Nidorellia armata & 0.05 & - & - & - & - & - & 0.05 & $0.05 \pm 0.00$ & - \\
\hline Pharia pyramidata & 0.05 & - & - & - & 0.05 & 0.05 & 0.10 & - & - \\
\hline Phataria unifascialis & - & $0.07 \pm 0.03$ & 0.05 & 0.10 & $0.13 \pm 0.04$ & 0.05 & 0.05 & 0.05 & - \\
\hline \multicolumn{10}{|l|}{ Clase Echinoidea } \\
\hline Astropyga pulvinata & - & 0.05 & - & - & - & - & - & - & - \\
\hline Diadema mexicanum & $4.78 \pm 1.12$ & $9.73 \pm 6.43$ & $6.02 \pm 5.65$ & $15.92 \pm 2.92$ & $217.08 \pm 2.43$ & $4.37 \pm 1.12$ & $7.67 \pm 4.47$ & $13.82 \pm 4.4$ & $5.08 \pm 2.58$ \\
\hline Eucidaris thouarsii & $0.30 \pm 0.30$ & $0.12 \pm 0.06$ & $0.70 \pm 0.26$ & $0.47 \pm 0.47$ & $0.42 \pm 0.38$ & $1.60 \pm 0.79$ & $0.27 \pm 0.21$ & $0.48 \pm 0.45$ & $0.28 \pm 0.11$ \\
\hline Toxopneustes roseus & - & $0.17 \pm 0.16$ & $0.27 \pm 0.10$ & $0.42 \pm 0.23$ & $0.08 \pm 0.04$ & - & - & $0.38 \pm 0.24$ & - \\
\hline Tripneustes depressus & - & - & - & - & - & $0.10 \pm 0.00$ & - & - & - \\
\hline \multicolumn{10}{|l|}{ Clase Holothuroidea } \\
\hline Chiridota rigida & - & - & - & - & - & - & 0.05 & - & - \\
\hline Euapta godeffroyi & $0.05 \pm 0.00$ & - & - & - & - & - & - & - & - \\
\hline Holothuria (Cystipus) casoae & - & - & - & - & - & - & 0.05 & 0.15 & - \\
\hline Holothuria (Mertensiothuria) hilla & - & - & - & - & 0.05 & 0.05 & $0.20 \pm 0.14$ & 0.15 & - \\
\hline Holothuria (Thymiosycia) arenicola & - & - & - & - & 0.05 & - & - & $0.13 \pm 0.14$ & - \\
\hline Isostichopus fuscus & - & 0.05 & 0.05 & - & - & - & - & - & - \\
\hline \multicolumn{10}{|l|}{ Filo Arthropoda } \\
\hline \multicolumn{10}{|l|}{ Orden Decapoda } \\
\hline Panulirus gracilis & - & 0.05 & - & - & - & - & - & - & - \\
\hline Stenorhynchus debilis & - & $0.10 \pm 0.00$ & $0.25 \pm 0.26$ & - & 0.05 & $0.13 \pm 0.04$ & - & $0.05 \pm 0.00$ & - \\
\hline \multicolumn{10}{|l|}{ Filo Mollusca } \\
\hline \multicolumn{10}{|l|}{ Clase Bivalvia } \\
\hline Pinctada mazatlanica & 0.05 & 0.15 & $0.05 \pm 0.00$ & 0.05 & $0.08 \pm 0.04$ & $0.05 \pm 0.00$ & 0.05 & $0.13 \pm 0.04$ & $0.20 \pm 0.21$ \\
\hline Pinna rugosa & - & - & - & - & 0.05 & - & 0.05 & - & - \\
\hline Spondylus spp. & - & - & - & 0.05 & $0.08 \pm 0.04$ & 0.05 & - & - & 0.20 \\
\hline \multicolumn{10}{|l|}{ Clase Cephalopoda } \\
\hline Octopus spp & - & - & - & - & - & 0.10 & - & - & - \\
\hline \multicolumn{10}{|l|}{ Filo Mollusca } \\
\hline \multicolumn{10}{|l|}{ Clase Gastropoda } \\
\hline Hexaplex princeps & - & - & 0.05 & 0.05 & - & $0.05 \pm 0.00$ & - & - & - \\
\hline Murex spp. & - & - & - & - & - & - & - & $0.05 \pm 0.00$ & - \\
\hline Muricidae spp. & - & - & - & 0.05 & 0.10 & - & - & - & $0.15 \pm 0.00$ \\
\hline \multicolumn{10}{|l|}{ Infraclase Opistobranchia } \\
\hline Dolabella spp. & 0.05 & - & - & - & - & - & - & - & - \\
\hline Elysia diomedea & - & - & - & - & - & - & $0.20 \pm 0.21$ & - & - \\
\hline Especie & 21 & 23 & 25 & 26 & 30 & 33 & 37 & 81 & 82 \\
\hline \multicolumn{10}{|l|}{ Filo Echinodermata } \\
\hline \multicolumn{10}{|l|}{ Clase Asteroidea } \\
\hline Pharia pyramidata & - & $0.05 \pm 0.03$ & - & - & - & - & - & - & - \\
\hline Phataria unifascialis & - & 0.05 & - & $0.08 \pm 0.04$ & - & 0.10 & - & $0.05 \pm 0.00$ & $0.08 \pm 0.04$ \\
\hline
\end{tabular}




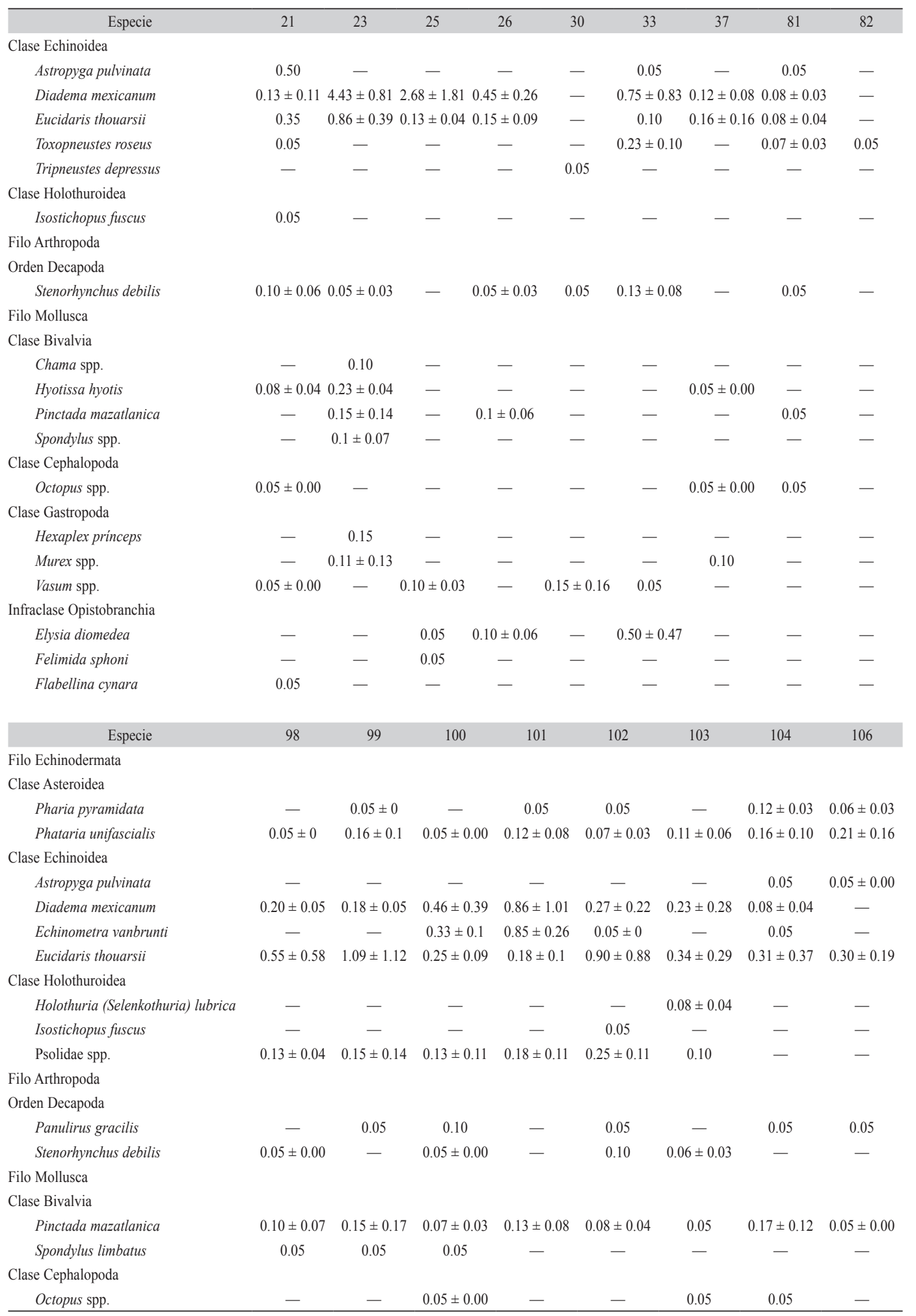




\begin{tabular}{|c|c|c|c|c|c|c|c|c|}
\hline Especie & 98 & 99 & 100 & 101 & 102 & 103 & 104 & 106 \\
\hline \multicolumn{9}{|l|}{ Clase Gastropoda } \\
\hline Fasciolariidae spp. & - & - & - & - & $0.05 \pm 0.05$ & - & - & - \\
\hline Hexaplex princeps & 0.05 & $0.10 \pm 0.07$ & - & $0.05 \pm 0.00$ & 0.05 & 0.05 & - & - \\
\hline Lobatus galeatus & - & - & - & - & 0.15 & - & - & - \\
\hline Murex spp. & - & - & - & - & - & 0.05 & - & - \\
\hline Muricidae spp. & 0.05 & 0.15 & 0.05 & 0.10 & $0.10 \pm 0.07$ & $0.15 \pm 0.07$ & - & - \\
\hline Vasum spp.. & - & 0.10 & - & $0.13 \pm 0.04$ & 0.05 & - & $0.15 \pm 0.13$ & - \\
\hline \multicolumn{9}{|l|}{ Infraclase Opistobranchia } \\
\hline Doriprismatica sedna & - & - & 0.05 & - & - & - & - & - \\
\hline Elysia diomedea & - & $0.08 \pm 0.03$ & 0.05 & $0.05 \pm 0.00$ & $0.05 \pm 0.00$ & $0.08 \pm 0.04$ & - & - \\
\hline Elysia spp. & $0.05 \pm 0.00$ & - & $0.05 \pm 0.00$ & - & $0.05 \pm 0.00$ & 0.05 & $0.05 \pm 0.00$ & - \\
\hline Felimare agassizii & $0.08 \pm 0.05$ & $0.05 \pm 0.00$ & $0.07 \pm 0.03$ & - & - & - & - & - \\
\hline Flabellina marcusorum & - & $0.07 \pm 0.03$ & - & - & - & - & - & - \\
\hline Otros opistobranquios & - & 0.05 & $0.25 \pm 0.09$ & 0.05 & $0.12 \pm 0.12$ & - & $0.08 \pm 0.04$ & - \\
\hline \multicolumn{2}{|l|}{ Especie } & 121 & \multicolumn{2}{|l|}{122} & 123 & \multicolumn{2}{|l|}{124} & 125 \\
\hline \multicolumn{9}{|l|}{ Filo Echinodermata } \\
\hline \multicolumn{9}{|l|}{ Clase Asteroidea } \\
\hline Nidorellia armata & & - & - & & 0.05 & \multirow{2}{*}{\multicolumn{2}{|c|}{$\frac{-}{0.05}$}} & - \\
\hline Phataria unifascialis & & - & - & & $0.05 \pm 0.00$ & & & - \\
\hline Clase Echinoidea & & & & & & & & \\
\hline Diadema mexicanum & & - & 0.05 & & $0.08 \pm 0.04$ & $0.83 \pm 1.03$ & & $0.05 \pm 0.00$ \\
\hline Echinometra vanbrunti & & 0.15 & - & & $0.08 \pm 0.04$ & - & & - \\
\hline Eucidaris thouarsii & & - & $0.10 \pm 0$. & 0.09 & $1.17 \pm 0.34$ & $0.24 \pm 0.23$ & & $0.15 \pm 0.12$ \\
\hline Clase Holothuroidea & & & & & & & & \\
\hline Holothuria (Halodeima) atra & & - & - & & - & 0.05 & & - \\
\hline Isostichopus fuscus & & - & - & & 0.15 & 0.05 & & 0.05 \\
\hline Psolidae spp. & & - & - & & 0.05 & - & & - \\
\hline Filo Arthropoda & & & & & & & & \\
\hline Orden Decapoda & & & & & & & & \\
\hline Panulirus gracilis & & $0.08 \pm 0.04$ & - & & - & - & & - \\
\hline Stenorhynchus debilis & & 0.10 & - & & $0.07 \pm 0.03$ & $0.13 \pm 0.11$ & & 0.10 \\
\hline Filo Mollusca & & & & & & & & \\
\hline Clase Bivalvia & & & & & & & & \\
\hline Atrina spp. & & - & - & & - & 0.10 & & - \\
\hline Hyotissa hyotis & & 0.05 & - & & 0.05 & - & & - \\
\hline Pinctada mazatlanica & & - & - & & $0.30 \pm 0.18$ & $0.41 \pm 0.21$ & & $0.15 \pm 0.10$ \\
\hline Pinna rugosa & & - & - & & 0.05 & - & & - \\
\hline Spondylus limbatus & & - & - & & $0.15 \pm 0.14$ & $0.30 \pm 0.14$ & & - \\
\hline Spondylus spp. & & - & - & & - & $0.10 \pm 0.07$ & & - \\
\hline Clase Gastropoda & & & & & & & & \\
\hline Hexaplex princeps & & - & - & & $0.13 \pm 0.11$ & $0.08 \pm 0.03$ & & 0.05 \\
\hline Vasum spp. & & - & - & & 0.05 & - & & - \\
\hline Infraclase Opistobranchia & & & & & & & & \\
\hline Elysia diomedea & & - & $0.18 \pm 0.1$ & 0.12 & - & - & & - \\
\hline Elysia spp. & & - & $0.05 \pm 0$. & 0.00 & - & - & & - \\
\hline Felimare agassizii & & - & $0.12 \pm 0.1$ & .12 & - & - & & - \\
\hline Felimida dalli & & - & - & & 0.05 & - & & - \\
\hline Flabellina marcusorum & & - & - & & 0.05 & - & & - \\
\hline Otros opistobranquios & & - & - & & 0.05 & 0.10 & & 0.05 \\
\hline
\end{tabular}




\section{ANEXO 2}

Densidades promedio ( \pm ds) de las especies de peces arrecifales en el Pacífico Norte de Costa Rica

Average densities $( \pm \mathrm{sd})$ of reef fish species in the North Pacific of Costa Rica

\begin{tabular}{|c|c|c|c|c|c|c|c|c|c|c|}
\hline Especie & 11 & 12 & 13 & 14 & 15 & 16 & 17 & 18 & 20 & 21 \\
\hline \multicolumn{11}{|l|}{ Orden Anguilliformes } \\
\hline \multicolumn{11}{|l|}{ Familia Muraenidae } \\
\hline Gymnothorax castaneus & - & - & - & - & $0.03 \pm 0.01$ & - & - & - & - & - \\
\hline \multicolumn{11}{|l|}{ Orden Aulopiformes } \\
\hline \multicolumn{11}{|l|}{ Familia Synodonthidae } \\
\hline Synodus lacertinus & - & - & $0.02 \pm 0.00$ & - & - & - & - & - & - & - \\
\hline \multicolumn{11}{|l|}{ Orden Beryciformes } \\
\hline \multicolumn{11}{|l|}{ Familia Apogonidae } \\
\hline Apogon dovii & - & - & $0.20 \pm 0.00$ & $0.33 \pm 0.23$ & - & - & - & $0.27 \pm 0.09$ & - & - \\
\hline \multicolumn{11}{|l|}{ Familia Holocentridae } \\
\hline Sargocentron suborbitalis & - & - & - & - & $0.19 \pm 0.15$ & $0.10 \pm 0.08$ & - & - & - & - \\
\hline \multicolumn{11}{|l|}{ Orden Perciformes } \\
\hline \multicolumn{11}{|l|}{ Familia Acanthuridae } \\
\hline Prionurus laticlavius & - & - & - & - & - & - & - & - & $1.05 \pm 1.06$ & - \\
\hline \multicolumn{11}{|l|}{ Familia Blenniidae } \\
\hline Ophioblennius steindachneri & - & - & - & - & - & - & - & - & $0.33 \pm 0.38$ & - \\
\hline Plagiotremus azaleus & - & - & - & - & - & - & - & - & - & $0.02 \pm 0.00$ \\
\hline \multicolumn{11}{|l|}{ Familia Carangidae } \\
\hline Caranx caballus & $0.03 \pm 0.01$ & - & $0.24 \pm 0.30$ & - & $0.09 \pm 0.01$ & - & - & - & - & $0.32 \pm 0.39$ \\
\hline \multicolumn{11}{|l|}{ Familia Chaetodontidae } \\
\hline Johnrandallia nigrirostris & - & $0.23 \pm 0.26$ & $0.10 \pm 0.13$ & - & $0.04 \pm 0.58$ & - & - & - & - & - \\
\hline \multicolumn{11}{|l|}{ Familia Cirrhitidae } \\
\hline Cirrhitichthys oxycephalus & $0.03 \pm 0.01$ & - & - & - & - & - & - & - & - & - \\
\hline \multicolumn{11}{|l|}{ Familia Haemulidae } \\
\hline Haemulon maculicauda & - & - & - & - & $0.50 \pm 0.26$ & - & - & - & $2.10 \pm 2.68$ & - \\
\hline Haemulon steindachneri & - & - & - & - & $0.03 \pm 0.01$ & - & - & - & - & - \\
\hline \multicolumn{11}{|l|}{ Familia Labridae } \\
\hline Bodianus diplotaenia & - & - & - & - & $0.02 \pm 0.01$ & $0.06 \pm 0.04$ & - & - & - & - \\
\hline Halichoeres chierchiae & - & - & - & - & - & - & - & - & - & - \\
\hline Halichoeres dispilus & - & $0.29 \pm 0.10$ & $0.07 \pm 0.04$ & - & - & $1.00 \pm 0.44$ & - & $0.60 \pm 0.56$ & - & $0.10 \pm 0.04$ \\
\hline Halichoeres notospilus & - & - & - & - & - & $0.12 \pm 0.00$ & - & - & - & - \\
\hline Thalassoma lucasanum & $1.11 \pm 0.50$ & $0.86 \pm 0.11$ & $1.22 \pm 0.18$ & - & - & $0.15 \pm 0.19$ & - & $0.29 \pm 0.12$ & $9.00 \pm 1.41$ & $0.61 \pm 0.45$ \\
\hline \multicolumn{11}{|l|}{ Familia Mullidae } \\
\hline Mulloidichthys dentatus & - & $0.07 \pm 0.04$ & - & - & - & - & - & - & - & - \\
\hline \multicolumn{11}{|l|}{ Familia Pomacanthidae } \\
\hline Holacanthus passer & $0.06 \pm 0.00$ & $0.06 \pm 0.00$ & $0.08 \pm 0.00$ & $0.02 \pm 0.00$ & - & - & $0.06 \pm 0.02$ & $0.02 \pm 0.00$ & - & $0.03 \pm 0.01$ \\
\hline Pomacanthus zonipectus & - & - & - & - & - & - & - & - & - & $0.03 \pm 0.01$ \\
\hline \multicolumn{11}{|l|}{ Familia Pomacentridae } \\
\hline Abudefduf troschelii & $0.20 \pm 0.14$ & - & $0.87 \pm 0.66$ & $0.26 \pm 0.05$ & $0.80 \pm 0.20$ & - & $0.23 \pm 0.05$ & - & - & - \\
\hline Chromis atrilobata & $4.13 \pm 3.80$ & $5.00 \pm 2.82$ & $2.26 \pm 3.23$ & $4.81 \pm 4.84$ & - & - & - & $2.13 \pm 1.80$ & $3.01 \pm 4.22$ & $5.00 \pm 1.41$ \\
\hline Microspathodon dorsalis & - & $0.07 \pm 0.05$ & - & - & - & - & - & $0.02 \pm 0$ & - & - \\
\hline Stegastes acapulcoensis & $0.50 \pm 0.1$ & $0.40 \pm 0.14$ & $0.20 \pm 0.14$ & $0.22 \pm 0.22$ & $0.45 \pm 0.39$ & $0.74 \pm 0.45$ & $0.26 \pm 0.05$ & $1.60 \pm 0.91$ & - & - \\
\hline Stegastes flavilatus & - & - & - & - & $0.35 \pm 0.21$ & $0.35 \pm 0.07$ & - & - & - & - \\
\hline
\end{tabular}

Familia Scaridae 


\begin{tabular}{|c|c|c|c|c|c|c|c|c|c|c|}
\hline Scarus ghobban & $0.37 \pm 0.30$ & $0.40 \pm 0.10$ & $0.43 \pm 0.15$ & $0.88 \pm 0.85$ & - & - & $1.88 \pm 0.39$ & $0.71 \pm 0.47$ & - & $0.10 \pm 0.06$ \\
\hline Scarus perrico & - & - & - & - & - & - & - & - & - & - \\
\hline Scarus rubroviolaceus & - & $0.17 \pm 0.12$ & - & - & - & - & - & $0.06 \pm 0.02$ & - & - \\
\hline \multicolumn{11}{|l|}{ Familia Serranidae } \\
\hline Alphestes multiguttatus & - & - & - & - & $0.02 \pm 0.00$ & - & - & - & - & - \\
\hline Cephalopholis panamensis & - & - & - & - & $0.08 \pm 0.08$ & - & - & - & $0.04 \pm 0.02$ & - \\
\hline Epinephelus labriformis & - & - & - & - & $0.04 \pm 0.03$ & - & - & - & $0.09 \pm 0.07$ & - \\
\hline Serranus psittacinus & - & $0.02 \pm 0.00$ & - & - & - & - & - & $0.22 \pm 0.02$ & - & $0.25 \pm 0.14$ \\
\hline \multicolumn{11}{|l|}{ Orden Syngnathiformes } \\
\hline \multicolumn{11}{|l|}{ Familia Fistulariidae } \\
\hline Fistularia commersonii & - & - & $0.09 \pm 0.09$ & $0.04 \pm 0.02$ & $0.02 \pm 0.00$ & - & $0.07 \pm 0.04$ & - & - & - \\
\hline \multicolumn{11}{|l|}{ Orden Tetraodontiformes } \\
\hline \multicolumn{11}{|l|}{ Familia Balistidae } \\
\hline Pseudobalistes naufragium & - & $0.04 \pm 0.00$ & - & $0.02 \pm 0.00$ & - & - & - & - & - & - \\
\hline Sufflamen verres & $0.25 \pm 0.13$ & $0.16 \pm 0.05$ & $5 \quad 0.23 \pm 0.24$ & $0.15 \pm 0.08$ & $0.09 \pm 0.04$ & $0.08 \pm 0.05$ & $0.07 \pm 0.01$ & $0.20 \pm 0.15$ & - & $0.15 \pm 0.10$ \\
\hline \multicolumn{11}{|l|}{ Familia Diodontidae } \\
\hline Diodon holocanthus & - & $0.18 \pm 0.22$ & - & $0.04 \pm 0.00$ & $0.14 \pm 0.18$ & - & - & $0.05 \pm 0.04$ & - & $0.03 \pm 0.02$ \\
\hline \multicolumn{11}{|l|}{ Familia Tetraodontidae } \\
\hline Canthigaster punctatissima & $0.04 \pm 0.00$ & $0.08 \pm 0.05$ & - & $0.05 \pm 0.04$ & $0.03 \pm 0.01$ & - & - & $0.02 \pm 0.00$ & - & $0.06 \pm 0.01$ \\
\hline Especie & 23 & 25 & 26 & 30 & 33 & 37 & 81 & 98 & 99 & 100 \\
\hline \multicolumn{11}{|l|}{ Orden Beryciformes } \\
\hline \multicolumn{11}{|l|}{ Familia Holocentridae } \\
\hline Sargocentron suborbitalis & $0.05 \pm 0.03$ & - & - & - & - & - & - & - & - & - \\
\hline \multicolumn{11}{|l|}{ Orden Perciformes } \\
\hline \multicolumn{11}{|l|}{ Familia Acanthuridae } \\
\hline Acanthurus xanthopterus & $0.23 \pm 0.12$ & - & - & - & - & - & - & - & - & - \\
\hline \multicolumn{11}{|l|}{ Familia Blenniidae } \\
\hline Plagiotremus azaleus & - & - & - & - & - & - & - & $0.04 \pm 0.02$ & $0.06 \pm 0.02$ & $0.04 \pm 0.03$ \\
\hline Familia Carangidae & - & - & - & - & - & - & - & - & - & - \\
\hline Gnathanodon speciosus & $0.12 \pm 0.05$ & - & - & - & - & - & - & - & - & - \\
\hline \multicolumn{11}{|l|}{ Familia Chaetodontidae } \\
\hline Chaetodon humeralis & - & - & $0.07 \pm 0.07$ & - & - & - & - & - & $0.02 \pm 0$ & $0.03 \pm 0.01$ \\
\hline Johnrandallia nigrirostris & $0.03 \pm 0.01$ & - & $0.05 \pm 0.02$ & - & $0.04 \pm 6.58$ & - & - & - & - & - \\
\hline \multicolumn{11}{|l|}{ Familia Cirrhitidae } \\
\hline Cirrhitichthys oxycephalus & - & - & $0.02 \pm 0.00$ & - & - & - & - & - & - & - \\
\hline Cirrhitus rivulatus & - & - & - & - & - & - & - & - & - & $0.02 \pm 0.00$ \\
\hline \multicolumn{11}{|l|}{ Familia Haemulidae } \\
\hline Anisotremus caesius & - & - & - & - & - & - & - & $0.04 \pm 0.00$ & $0.02 \pm 0.00$ & - \\
\hline Haemulon flaviguttatum & - & - & - & - & - & - & - & $0.05 \pm 0.01$ & $0.08 \pm 0.05$ & - \\
\hline Haemulon maculicauda & - & - & $0.50 \pm 0.40$ & - & - & - & - & $3.00 \pm 1.73$ & - & - \\
\hline \multicolumn{11}{|l|}{ Familia Kyphosidae } \\
\hline Kiphosus sp. & $0.17 \pm 0.04$ & - & - & - & - & - & - & - & - & - \\
\hline Kyphosus analogus & $0.22 \pm 0.02$ & - & - & - & - & - & - & - & - & - \\
\hline Kyphosus elegans & $0.12 \pm 0.11$ & - & - & - & - & - & - & - & - & - \\
\hline \multicolumn{11}{|l|}{ Familia Labridae } \\
\hline Bodianus diplotaenia & $0.03 \pm 0.01$ & - & - & - & - & - & - & - & $0.32 \pm 0.41$ & - \\
\hline Halichoeres dispilus & - & $0.06 \pm 0.05$ & - & - & $0.68 \pm 1.21$ & $0.05 \pm 0.01$ & - & $0.41 \pm 0.34$ & $0.23 \pm 0.24$ & $0.38 \pm 0.40$ \\
\hline Halichoeres nicholsi & - & - & - & - & - & - & - & $0.02 \pm 0.00$ & - & $0.03 \pm 0.01$ \\
\hline Halichoeres notospilus & - & - & - & - & - & - & $0.07 \pm 0.04$ & - & $0.04 \pm 0.00$ & $0.29 \pm 0.10$ \\
\hline Thalassoma lucasanum & $0.20 \pm 0.16 \quad 0$ & $0.34 \pm 0.38$ & $0.28 \pm 0.25$ & - & $0.36 \pm 0.33$ & $1.16 \pm 0.77$ & - & $0.15 \pm 0.09$ & $0.67 \pm 0.59$ & $0.74 \pm 0.53$ \\
\hline
\end{tabular}




\section{Familia Lutjanidae}

Hoplopagrus guentheri Lutjanus argentiventris

Lutjanus guttatus

Lutjanus inermis

Lutjanus novemfasciatus

Familia Malacanthidae

Malacanthus brevirostris

Familia Pomacanthidae

Holacanthus passer

Familia Pomacentridae

Abudefduf concolor

Abudefduf troschelii

Chromis atrilobata

Microspathodon dorsalis

Stegastes acapulcoensis

Stegastes flavilatus

Familia Scaridae

Scarus ghobban

Familia Serranidae

Cephalopholis panamensis

Epinephelus labriformis

Serranus psittacinus

Orden Syngnathiformes

Familia Fistulariidae

Fistularia commersonii

Orden Tetraodontiformes

Familia Balistidae

Pseudobalistes naufragium

Sufflamen verres

Familia Diodontidae

Diodon holocanthus

Familia Tetraodontidae

Arothron meleagris

Canthigaster punctatissima

Sphoeroides lobatus

$$
\begin{array}{cccc}
0.09 \pm 0.08 & - & - & - \\
- & - & 0.58 \pm 0.43 & - \\
- & - & 0.13 \pm 0.06 & - \\
0.04 \pm 0.04 & - & - & - \\
- & - & - & 0.05 \pm 0.01
\end{array}
$$

$0.02 \pm 0.01$

$0.50 \pm 0.23$

$2.46 \pm 1.36$

$0.46 \pm 0.31$

-

$0.23 \pm 0.18$

$0.8 \pm 0$

$0.04 \pm 0.02 \quad 0.26 \pm 0.19$

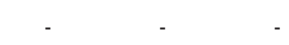

\section{Orden Perciformes}

Familia Acanthuridae

Prionurus laticlavius

Familia Blenniidae

Ophioblennius steindachneri

Plagiotremus azaleus

$0.03 \pm 0.01 \quad 0.06 \pm 9.31 \quad 0.03 \pm 0.02 \quad 0.03 \pm 0.01$

$0.04 \pm 0.00 \quad 0.04 \pm 0.02 \quad 0.05 \pm 0.01 \quad 0.02 \pm 0.00 \quad 0.04 \pm 0.00$

Chaetodon humeralis

Johnrandallia nigrirostris

Familia Haemulidae

Anisotremus caesius

Haemulon flaviguttatum
$0.02 \pm 0.01 \quad 0.02 \pm 0.01 \quad-$

$0.08 \pm 0.08$

$0.27 \pm 0.24$
$-$

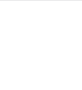

- $\quad-\quad-\quad 0.05 \pm 0.01$

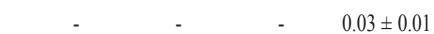

$\begin{array}{llll}0.02 \pm 0.00 \quad- & 0.03 \pm 0.01 & 0.02 \pm 0.01\end{array}$

$0.17 \pm 0.22 \quad 0.06 \pm 0.02 \quad-\quad 0.03 \pm 0.01$ $0.05 \pm 0.04 \quad 0.14 \pm 0.15 \quad 0.03 \pm 0.01$

$0.02 \pm 0.00$

$0.09 \pm 0.09$ 


\section{Familia Labridae}

Bodianus diplotaenia Halichoeres chierchiae Halichoeres dispilus Halichoeres nicholsi Halichoeres notospilus Thalassoma lucasanum Familia Lutjanidae

Lutjanus guttatus

Familia Pomacanthidae

Holacanthus passer

Familia Pomacentridae

Abudefduf troschelii

Chromis atrilobata

Microspathodon dorsalis

Stegastes acapulcoensis

Stegastes flavilatus

Familia Scaridae

Scarus ghobban

Familia Serranidae

Alphestes immaculatus

Cephalopholis panamensi

Epinephelus labriformis

Serranus psittacinus

Orden Tetraodontiformes

Familia Balistidae

Sufflamen verres

Diodon holocanthus

Familia Tetraodontidae

Arothron hispidus

Arothron meleagris

Canthigaster punctatissima

\begin{tabular}{|c|c|c|c|c|c|c|c|c|c|c|}
\hline- & $0.22 \pm 0.23$ & $0.03 \pm 0.02$ & - & $0.10 \pm 0.08$ & - & - & - & $0.12 \pm 0.11$ & $0.08 \pm 0.05$ & $0.07 \pm 0.07$ \\
\hline - & - & - & - & - & - & - & - & $0.02 \pm 0.00$ & - & $0.03 \pm 0.01$ \\
\hline- & $0.28 \pm 0.35$ & $0.65 \pm 0.65$ & $0.29 \pm 0.24$ & $0.27 \pm 0.12$ & $0.42 \pm 0.27$ & $1.72 \pm 1.87$ & $0.19 \pm 0.21$ & - & $0.19 \pm 0.09$ & $0.60 \pm 0.87$ \\
\hline \pm 0.09 & $0.04 \pm 0.01$ & $0.12 \pm 0.17$ & - & - & - & - & $0.02 \pm 0.00$ & $0.03 \pm 0.01$ & - & $0.02 \pm 0.00$ \\
\hline .01 & $0.03 \pm 0.02$ & $0.07 \pm 0.04$ & - & - & - & - & - & - & - & $0.19 \pm 0.11$ \\
\hline 11 & $0.15 \pm 0.12$ & $0.35 \pm 0.38$ & - & - & - & - & $0.39 \pm 0.54$ & $0.08 \pm 0.03$ & $0.36 \pm 0.14$ & $0.18 \pm 0.18$ \\
\hline
\end{tabular}

$0.08 \pm 0.05 \quad 0.40 \pm 0.05 \quad 0.32 \pm 0.11$

$\begin{array}{ccc}- & 0.55 \pm 0.37 & 0.14 \pm 0.16 \\ - & 4.20 \pm 2.30 & 1.39 \pm 2.25 \\ - & 0.06 \pm 0.06\end{array}$

$0.18 \pm 0.14 \quad 0.23 \pm 0.18 \quad 0.14 \pm 0.08$

$\begin{array}{llllllllllll}0.07 & \pm 0.07 & 0.12 \pm 0.06 & 0.23 \pm 0.17 & 0.45 \pm 0.18 & 0.22 \pm 0.17 & 0.16 \pm 0.02 & - & 0.24 \pm 0.24 & 0.10 \pm 0.06 & 0.06 \pm 0.01 & 0.16 \pm 0.17\end{array}$

\begin{tabular}{|c|c|c|c|c|c|c|c|c|c|}
\hline - & - & - & - & - & - & $0.09 \pm 0.01$ & - & - & - \\
\hline - & - & - & - & - & - & $0.02 \pm 0.00$ & - & - & - \\
\hline $0.04 \pm 0.00$ & $0.02 \pm 0.01$ & - & $0.02 \pm 0.00$ & - & - & - & - & - & - \\
\hline $0.03 \pm 0.01$ & $0.02 \pm 0.00$ & $0.02 \pm 0.00$ & - & - & - & $0.04 \pm 0.02$ & $0.04 \pm 0.00$ & $0.04 \pm 0.02$ & $0.05 \pm 0.01$ \\
\hline- & $0.05 \pm 0.03$ & - & - & - & $0.08 \pm 0.03$ & $0.16 \pm 0.04$ & - & - & - \\
\hline $0.02 \pm 0.00$ & - & - & - & - & - & $0.13 \pm 0.09$ & $0.03 \pm 0.01$ & $0.02 \pm 3.29$ & $0.05 \pm 0.03$ \\
\hline - & - & - & - & - & - & $0.02 \pm 3.29$ & - & - & - \\
\hline - & - & - & - & - & - & - & - & - & $0.02 \pm 3.29$ \\
\hline - & - & - & - & - & - & - & - & - & $0.07 \pm 0.07$ \\
\hline- & $0.03 \pm 0.01$ & - & - & - & - & $0.06 \pm 0.02$ & - & $0.04 \pm 0.02$ & - \\
\hline
\end{tabular}

